\title{
New and little-known bees of the genus Sphecodes Latreille, 1804 (Hymenoptera: Apoidea: Halictidae) from the Himalayas
}

\author{
Yulia ASTAFUROVA ${ }^{1} \&$ Maxim PROSHCHALYKIN ${ }^{2, *}$ \\ ${ }^{1}$ Zoological Institute, Russian Academy of Sciences (ZISP), Saint Petersburg, Russia. \\ ${ }^{2}$ Federal Scientific Centre for East Asian Terrestrial Biodiversity, Far Eastern Branch of the Russian \\ Academy of Sciences (FCBV), Vladivostok, Russia. \\ *Corresponding author: proshchalikin@biosoil.ru \\ 1Email: Yulia.Astafurova@zin.ru \\ ${ }^{1}$ urn:lsid:zoobank.org:author:E0C904B0-6727-4F5C-8F5D-3CD1BFF32F3B \\ ${ }^{2}$ urn:lsid:zoobank.org:author:6B6EEC64-E26C-4E34-A0C9-8DC198B881ED
}

\begin{abstract}
An annotated list of twenty species of rarely collected and little known bees of the genus Sphecodes Latreille, 1804 (Hymenoptera: Apoidea: Halictidae) from the Himalayas is given. Sphecodes bluethgeni sp. nov. is described from Bhutan. Three species are newly recorded from the Himalayas: S. binghami Blüthgen, 1924, S. kershawi Perkins, 1921 and S. laticeps Meyer, 1920. Based on type specimens, new synonymies have been proposed for Sphecodes cameronii (Bingham, 1897) = S. armeniacus Warncke, 1992 syn. nov.; S. gibbus (Linnaeus, 1758) = S. indicus Bingham, 1898 syn. nov.; and S. invidus (Cameron, 1897) $=$ S. nigrobasalis Meyer, 1922 syn. nov. A lectotype is designated for Sphecodes sikkimensis Blüthgen, 1927. Illustrated keys to males and females of all species known from the Himalayas and an updated checklist of the 26 Himalayan species of Sphecodes are provided. Additionally, one new species from neighboring Uttar Pradesh (India), Sphecodes uttaricus sp. nov., is here described as new and included due to its close relationship to the Himalayan S. sikkimensis Blüthgen, 1927.
\end{abstract}

Keywords. Taxonomy, new species, new synonyms, fauna, Oriental region.

Astafurova Y. \& Proshchalykin M. 2020. New and little-known bees of the genus Sphecodes Latreille, 1804 (Hymenoptera: Apoidea: Halictidae) from the Himalayas. European Journal of Taxonomy 729: 74-120. https://doi.org/10.5852/ejt.2020.729.1195

\section{Introduction}

The cleptoparasitic genus Sphecodes Latreille, 1804, numbering 70 species in the Palaearctic Region and 50 species in the Oriental Region, has long been one of the least studied groups in the family Halictidae Thomson, 1869. Previous reviews of some regional faunas (Central and Southeast Asia, Mongolia, China, Turkey, Iran, Caucasus, Near East, Central Europe, etc.) have significantly improved our knowledge of these bees, helping bridge the gaps in our knowledge of Sphecodes in these territories. The species composition of these faunas has been greatly clarified, twelve new species have been 
described, seventeen new synonymies have been proposed, and the taxonomical status of some species has been revised (Bogusch \& Straka 2012; Astafurova \& Proshchalykin 2014, 2015a, 2015b, 2015c, 2016a, 2016b, 2017a, 2017b, 2018; Astafurova et al. 2015, 2018a, 2018b, 2018c, 2018d, 2019, 2020b; Özbek et al. 2015).

However, the territories located in the transitional zone between the Palaearctic and the Oriental regions remain insufficiently studied. The Himalayas are the perfect example of this situation. Geographically, the Himalayas are located in the Indian subcontinent. The countries and territories included are: India (Arunachal Pradesh, Himachal Pradesh, Jammu and Kashmir, Meghalaya, Mizoram, Nagaland, Sikkim, Tripura, Uttarakhand, Darjeeling district of West Bengal), Tajikistan (Gorno-Badakhshan Province), Afghanistan (Badakhshan Province), Bhutan, Nepal, China (Aksai Chin, Xizang), Myanmar (Kachin State), and Pakistan (Azad Jammu and Kashmir, Gilgit-Baltistan, Khyber Pakhtunkhwa) (Fig. 1). The bee fauna of the Himalayas is generally under-recorded and, given the enormous size and ecological diversity of the area, the discovery of large numbers of undescribed species including endemics is very

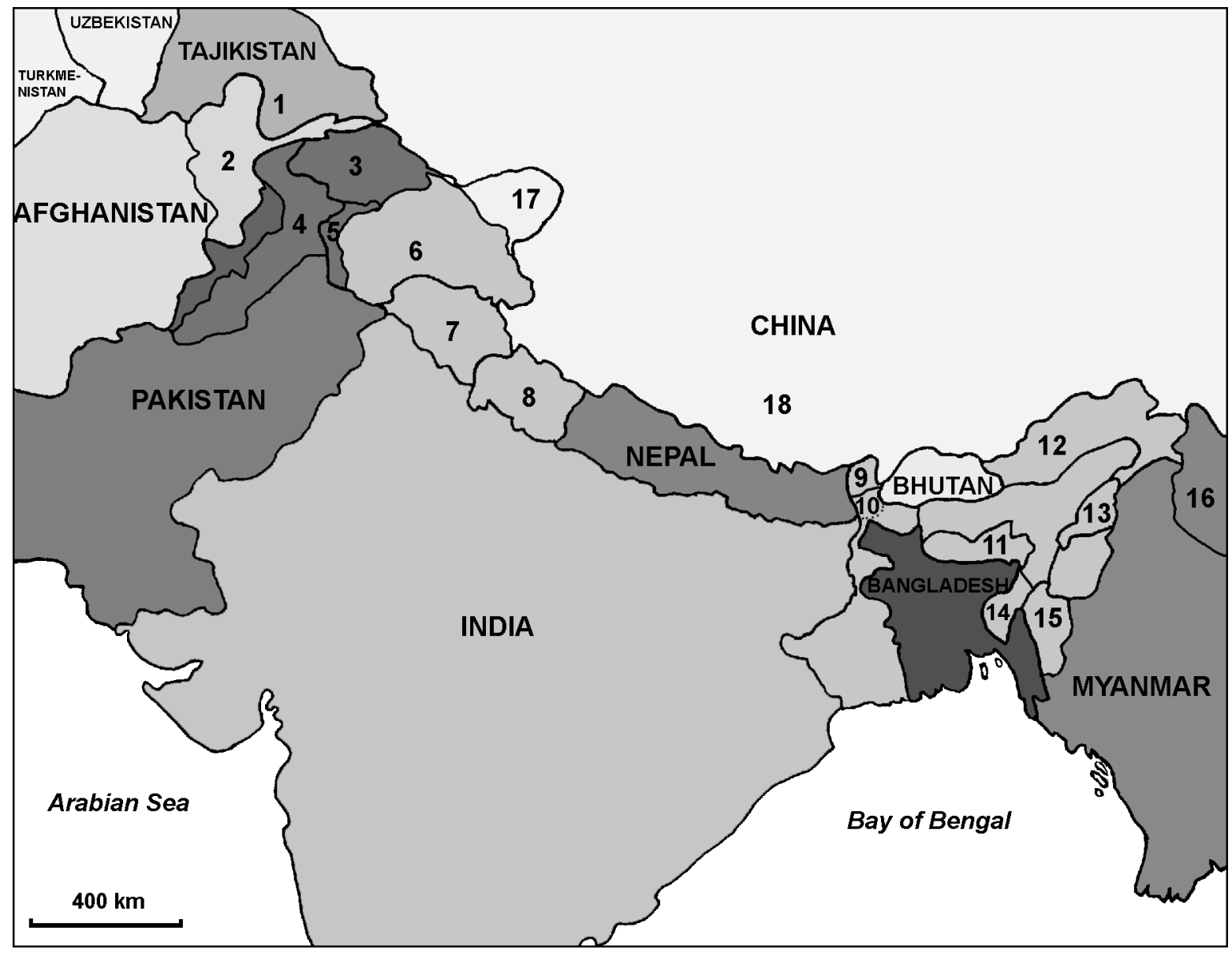

Fig. 1. Geographic map of the Himalayas and surrounding territories. $1=$ Gorno-Badakhshan Province (Tajikistan); 2 = Badakhshan Province (Afghanistan); 3 = Gilgit-Baltistan; 4 = Khyber Pakhtunkhwa; $5=$ Azad Jammu and Kashmir (Pakistan); $6=$ Jammu and Kashmir; $7=$ Himachal Pradesh; $8=$ Uttarakhand; $9=$ Sikkim; $10=$ Darjeeling district of West Bengal; $11=$ Meghalaya; $12=$ Arunachal Pradesh; $13=$ Nagaland; 14 = Tripura; $15=$ Mizoram (India); $16=$ Kachin State (Myanmar); $17=$ Aksai Chin; $18=$ Xizang (China). 
likely. Thus, the goal of this paper is to improve our knowledge of the taxonomy and distribution of Himalayan Sphecodes bees as an essential foundation for more advanced biogeographical investigations.

The first information on the genus Sphecodes from the Himalayas was published by Smith (1853), who described S. fumipennis from "N. India". This species has since only been recorded from Sikkim (Blüthgen 1924, 1927; Saini \& Rathor 2012; Rajkumar \& Dey 2016). A total of nineteen species have been described since from the Himalayas (Blüthgen -6 species, Nurse -4 species, Smith -3 species, Cameron -2 species, Bingham - 2, Cockerell - 1, and Gupta \& Saini - 1), with fourteen of them still valid (see section on taxonomy for details). In total, twenty three species of Sphecodes have been recorded from the Himalayas so far (Table 1).

In the present paper, based on a comprehensive study of specimens (including types) deposited in various collections, we report additional records of 20 species from this area. Further, one species is described as new and three species are recorded from the Himalayas for the first time, resulting in a total number of 26 species of Sphecodes known from this region (Table 1). The genus Sphecodes has not yet been documented from the Badakhshan Province of Afghanistan, Kachin State of Myanmar or Chinese Aksai Chin and Xizang, but it is probable that this cosmopolitan genus is present in these territories and it is only a matter of time before their faunas are sampled and recorded. A key to all species occurring in the Himalayas is also presented.

In addition, we describe a new species from neighboring Uttar Pradesh (India), Sphecodes uttaricus sp. nov., included due to its close relationship to the Himalayan S. sikkimensis Blüthgen, 1927, propose new synonymies for three specific names (Sphecodes cameronii (Bingham, 1897) $=S$. armeniacus Warncke, 1992 syn. nov.; S. gibbus (Linnaeus, 1758) = S. indicus Bingham, 1898 syn. nov.; and S. invidus (Cameron, 1897) =S. nigrobasalis Meyer, 1922 syn. nov.), and designate a lectotype for Sphecodes sikkimensis Blüthgen, 1927 in order to clarify the status and diagnosis of type specimens.

\section{Material and methods}

The results presented in this paper are based on 66 specimens collected in the Himalayas and currently housed in the following depositories:

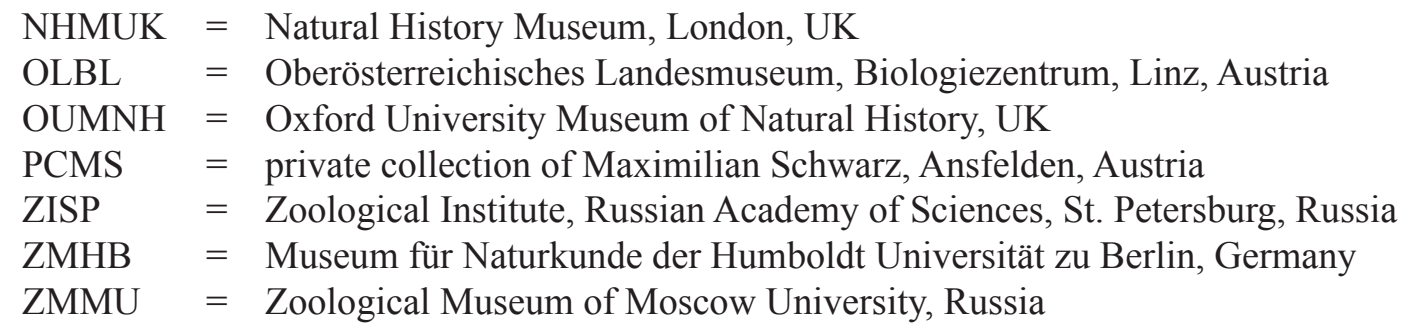

The taxonomy and distribution of species follows that of Warncke (1992), Astafurova \& Proshchalykin (2017b) and Astafurova et al. (2020a, 2020b). Morphological terminology follows that of Engel (2001) and Michener (2007). The ventral surface of some flagellomeres bears a distinctive patch of sensilla trichodea A (sensu Ågren \& Svensson 1982), which we refer to as 'tyloids', easily observable under the microscope. The abbreviations F, T and S are used for flagellomere, metasomal tergum and metasomal sternum respectively. The density of integumental punctures is described using the following formula: puncture diameter (in $\mu \mathrm{m}$ ) / ratio of distance between punctures to average puncture diameter, e.g., 15$20 \mu \mathrm{m} / 0.5-1.5$. Integumental sculpture other than distinctive surface punctation is described following Harris (1979): areolate - coarse, contiguous punctures; reticulate - superficially net-like or network of raised lines; rugose - irregular, nonparallel, wrinkled raised lines (rugae); rugulose - minutely rugose; 
Table 1. Checklist of the species of Sphecodes Latreille, 1804 of the Himalayas including distribution by countries and provinces. Abbreviations: $\mathrm{P}=$ Palaearctic species; $\mathrm{O}=$ Oriental species; $\mathrm{E}=$ endemic.

\begin{tabular}{|c|c|c|c|c|}
\hline $\mathbf{N}$ & Species of Sphecodes & Country & Published data & $\begin{array}{c}\text { Type of } \\
\text { area }\end{array}$ \\
\hline 1 & S. albilabris (Fabricius, 1793) & $\begin{array}{l}\text { India (*Himachal Pradesh), } \\
\text { *Nepal, Tajikistan (Gorno- } \\
\text { Badakhshan Province) }\end{array}$ & Astafurova \& Proshchalykin 2017b & $\mathrm{P}$ \\
\hline 2 & S. almoransis Gupta \& Saini, 2018 & India (Uttarakhand) & Gupta \& Saini 2018 & $\mathrm{E}$ \\
\hline 3 & S. alternatus Smith, 1853 & $\begin{array}{l}\text { Tajikistan (Gorno-Badakhshan } \\
\text { Province) }\end{array}$ & Astafurova \& Proshchalykin 2017b & $\mathrm{P}$ \\
\hline 4 & S. binghami Blüthgen, 1924 & *Nepal & first record & $\mathrm{O}$ \\
\hline 5 & S. bluethgeni sp. nov. & *Bhutan & first record & $\mathrm{E}$ \\
\hline 6 & S. cameronii (Bingham, 1897) & *Nepal, India (Uttarakhand) & Rajkumar \& Dey 2016 & $\mathrm{P}-\mathrm{O}$ \\
\hline 7 & S. dissimilandus (Cameron, 1897) & India (Uttarakhand) & $\begin{array}{l}\text { Cameron 1897; Saini \& Rathor 2012; } \\
\text { Rajkumar \& Dey } 2016\end{array}$ & $\mathrm{E}$ \\
\hline 8 & S. fumipennis Smith, 1853 & India (Sikkim) & $\begin{array}{l}\text { Blüthgen 1924, 1927; } \\
\text { Saini \& Rathor 2012; } \\
\text { Rajkumar \& Dey } 2016\end{array}$ & $\mathrm{O}$ \\
\hline 9 & S. gibbus (Linnaeus, 1758) & $\begin{array}{l}\text { India (Jammu and Kashmir, } \\
\text { Simla), Tajikistan (Gorno- } \\
\text { Badakhshan Province) }\end{array}$ & $\begin{array}{l}\text { Smith 1853; Bingham 1898; } \\
\text { Nurse 1903; Saini \& Rathor 2012; } \\
\text { Astafurova \& Proshchalykin 2017b }\end{array}$ & $\mathrm{P}$ \\
\hline 10 & S. hakkariensis Warncke, 1992 & $\begin{array}{l}\text { Tajikistan (Gorno-Badakhshan } \\
\text { Province) }\end{array}$ & Astafurova et al. 2018c & $\mathrm{P}$ \\
\hline 11 & S. intermedius Blüthgen, 1923 & $\begin{array}{l}\text { Tajikistan (Gorno-Badakhshan } \\
\text { Province) }\end{array}$ & Astafurova \& Proshchalykin 2017b & $\mathrm{P}$ \\
\hline 12 & S. invidus (Cameron, 1897) & India (Uttarakhand, Meghalaya) & Cameron 1897; Meyer 1922 & $\mathrm{E}$ \\
\hline 13 & S. iridipennis Smith, 1879 & $\begin{array}{l}\text { India (Uttarakhand, Himachal } \\
\text { Pradesh) }\end{array}$ & $\begin{array}{l}\text { Saini \& Rathor 2012; Gupta 2013; } \\
\text { Ascher \& Pickering } 2020\end{array}$ & E \\
\hline 14 & S. kershawi Perkins, 1921 & *India (Uttarakhand) & first record & $\mathrm{O}$ \\
\hline 15 & S. lasimensis Blüthgen, 1927 & $\begin{array}{l}\text { India (Himachal Pradesh), } \\
\text { *Nepal }\end{array}$ & $\begin{array}{l}\text { Blüthgen 1927; Saini \& Rathor 2012; } \\
\text { Rajkumar \& Dey 2016; } \\
\text { Ascher \& Pickering } 2020\end{array}$ & $\mathrm{E}$ \\
\hline 16 & S. laticeps Meyer, 1920 & *Nepal & first record & $\mathrm{O}$ \\
\hline 17 & S. monilicornis (Kirby, 1802) & $\begin{array}{l}\text { India (Jammu and Kashmir), } \\
\text { *Nepal, Tajikistan (Gorno- } \\
\text { Badakhshan Province) }\end{array}$ & $\begin{array}{l}\text { Nurse 1903; Blüthgen 1927; } \\
\text { Saini \& Rathor 2012; } \\
\text { Astafurova \& Proshchalykin } 2017 \text { b }\end{array}$ & $\mathrm{P}$ \\
\hline 18 & S. montanus Smith, 1879 & $\begin{array}{l}\text { India (Uttarakhand), *Nepal, } \\
\text { *Bhutan }\end{array}$ & Smith 1879; Rajkumar \& Dey 2016 & $\mathrm{O}$ \\
\hline 19 & S. perplexus Nurse, 1903 & India (Jammu and Kashmir) & Nurse 1903; Saini \& Rathor 2012 & $\mathrm{E}$ \\
\hline 20 & S. rufiventris (Panzer, 1798) & $\begin{array}{l}\text { Tajikistan (Gorno-Badakhshan } \\
\text { Province) }\end{array}$ & Astafurova \& Proshchalykin 2017b & $\mathrm{P}$ \\
\hline 21 & S. setiger Blüthgen, 1924 & India (Himachal Pradesh) & Blüthgen 1924 & $\mathrm{E}$ \\
\hline 22 & S. shillongensis Blüthgen, 1927 & India (Meghalaya) & $\begin{array}{l}\text { Saini \& Rathor 2012; } \\
\text { Ascher \& Pickering } 2020\end{array}$ & $\mathrm{E}$ \\
\hline 23 & S. sikkimensis Blüthgen, 1927 & India (Sikkim) & $\begin{array}{l}\text { Blüthgen 1927; Saini \& Rathor 2012; } \\
\text { Rajkumar \& Dey } 2016\end{array}$ & $\mathrm{O}$ \\
\hline 24 & S. simlaensis Blüthgen, 1924 & $\begin{array}{l}\text { India (Jammu and Kashmir, } \\
\text { Himachal Pradesh, } \\
\text { *Uttarakhand), *Nepal, *Bhutan }\end{array}$ & $\begin{array}{l}\text { Blüthgen 1924, 1927; } \\
\text { Saini \& Rathor 2012; } \\
\text { Rajkumar \& Dey 2016 }\end{array}$ & $\mathrm{O}$ \\
\hline 25 & S. tantalus Nurse, 1903 & India (Jammu and Kashmir) & $\begin{array}{l}\text { Nurse 1903; Saini \& Rathor 2012; } \\
\text { Dar \& Wani, 2018; Dar et al. } 2018\end{array}$ & $\mathrm{E}$ \\
\hline 26 & S. turneri Cockerell, 1916 & India (Meghalaya) & $\begin{array}{l}\text { Cockerell, 1916; } \\
\text { Ascher \& Pickering } 2020\end{array}$ & $\mathrm{O}$ \\
\hline
\end{tabular}


strigate - narrow, transverse or longitudinal streaks (strigae), variety of parallel lineations; tessellate regular network of shallow grooves with flat interspaces.

Specimens were studied with a Leica M205A stereo microscope and photographs taken with a combination of stereo microscope (Olympus SZX10) and digital camera (Olympus OM-D and Canon EOS70D). Final images are stacked composites using Helicon Focus 6. Specimen data and images of NHMUK specimens (Figs 7, 12-13) were recorded in the NHMUK database or on the NHMUK database website, and are publicly available through the NHMUK Data Portal (Natural History Museum 2014). All images were post-processed for contrast and brightness using Adobe Photoshop. New distributional records are noted with an asterisk (*).

\section{Results}

\section{Key to the species of Sphecodes of the Himalayas}

Males are unknown in S. almoransis, S. binghami, S. bluethgeni sp. nov., S. iridipennis, S. perplexus, S. setiger, S. tantalus, and females are unknown in $S$. dissimilandus, S. invidus, S. shillongensis.

\section{Males}

1. Costal margin of hind wing with 7-14 hamuli. Base of gonocoxite dorsally without impression. Usually larger species: total body length $6.0-14.0 \mathrm{~mm}$ 2

- Costal margin of hind wing with 5-6 hamuli. Base of gonocoxite dorsally with or without impression.

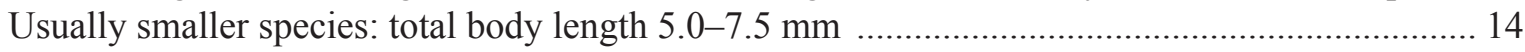

2. Gena flat. Preoccipital lateral carina well-developed .................................................................. 3

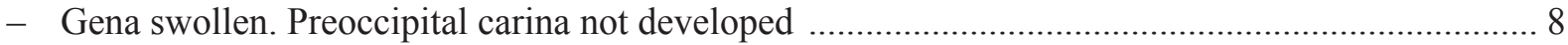

3. Fore wing with two cubital cells. Tyloids weakly developed, narrowly semicircular across at most $1 / 4$ of the basal flagellar surfaces (Fig. 2H). Body length 7.0-8.5 mm ..........S. turneri Cockerell, 1916

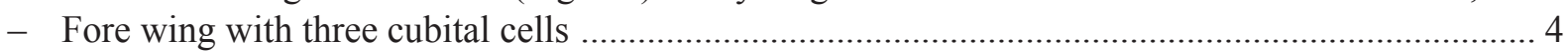

4. Tyloids covering entire ventral flagellar surface (Fig. 2G). Body length 7.0-8.5 mm

S. kershawi Perkins, 1921

- Ventral flagellar surface with medial glabrous spot or tyloids narrowly semicircular across basal flagellar surface 5

5. T1 with marginal zone impunctate. Body length $7.0-8.5 \mathrm{~mm}$......................S. laticeps Meyer, 1920

- T1 with marginal zone distinctly punctate (at least on a basal half) .............................................. 6

6. Wings with strong brownish darkening. Tyloids weakly developed, covering at most basal $1 / 4$ of ventral flagellar surface and narrowly linear across lateral surface (Fig. 2D). Large species: total body length more $12.0 \mathrm{~mm}$

S. sikkimensis Blüthgen, 1927

- Wings hyaline, without darkening. Tyloids well developed, covering entire lateral flagellar surface and peripheral part of ventral surface (with medial glabrous spot, variable in size). Smaller species: total body length $7.0-8.5 \mathrm{~mm}$

7. Vertex more elevated, distance from top of head to upper margin of lateral ocellus ca two lateral ocellar diameters as seen in frontal view. Ocello-ocular area with confluent punctures (areolatepunctate). Metasomal terga coarser punctate $(15-25 \mu \mathrm{m})$. Body length $7.5-8.5 \mathrm{~mm}$

S. invidus (Cameron, 1897)

- Vertex less elevated, distance from top of head to upper margin of lateral ocellus ca 1.5 lateral ocellar diameter as seen in frontal view. Ocello-ocular area with small shiny interspaces between 
punctures (at most a half puncture diameter). Metasomal terga finer punctate (10-20 $\mu \mathrm{m})$. Body length $7.0 \mathrm{~mm}$ S. dissimilandus (Cameron, 1897)

8. Head rounded, approximately as long as wide. Hind wing with basal vein strongly curved, the angle between basal $(M)$ and cubital $(\mathrm{Cu})$ veins ca $90^{\circ}$. T1 finely and sparsely (sometimes indistinctly) punctate. Gonostylus dorsally with small rectangular process directed to penis valve (Fig. 3B). Body length $7.0-10 \mathrm{~mm}$

S. monilicornis (Kirby, 1802)

- Head transverse, wider than long. Hind wing with basal vein weakly curved, the angle between basal $(\mathrm{M})$ and cubital $(\mathrm{Cu})$ veins $70-80^{\circ}$. T1 coarsely and densely punctate. Gonostylus of another shape .

9. Mesoscutum densely punctate, with confluent punctures (areolate-punctate) 10

- Mesoscutum sparser punctate, medially with punctures separated by at least a puncture diameter ..

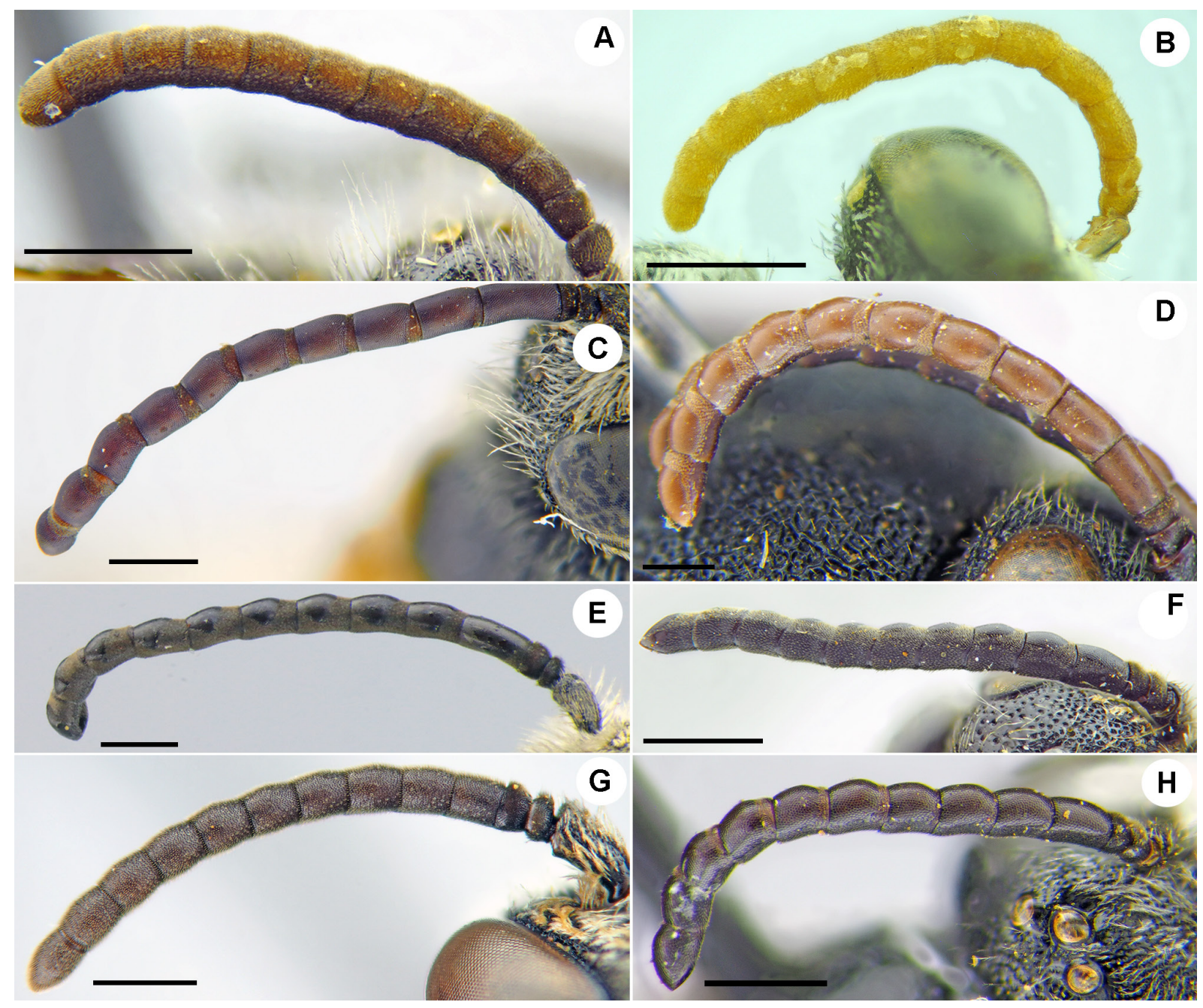

Fig. 2. Antennae in lateral view, males. A. Sphecodes hakkariensis Warncke, 1992. B. S. cameronii (Bingham, 1897). C. S. alternatus Smith, 1853. D. S. sikkimensis Blüthgen, 1927. E. S. gibbus (Linnaeus, 1758). F. S. rufiventris (Panzer, 1798). G. S. kershawi Perkins, 1921. H. S. turneri Cockerell, 1916. Scale bars $=0.5 \mathrm{~mm}$. 
10. Head more transverse, 1.25-1.3 times as wide as long. Gonostylus longer, apically elongated into rounded process with long setae (Fig. 3E). Body length 9.0-12.0 mm

S. albilabris (Fabricius, 1793)

- Head less transverse, ca 1.2 times as wide as long. Gonostylus short, not enlarged apically (Fig. 3A). Body length $9.5-12 \mathrm{~mm}$ S. fumipennis Smith, 1853
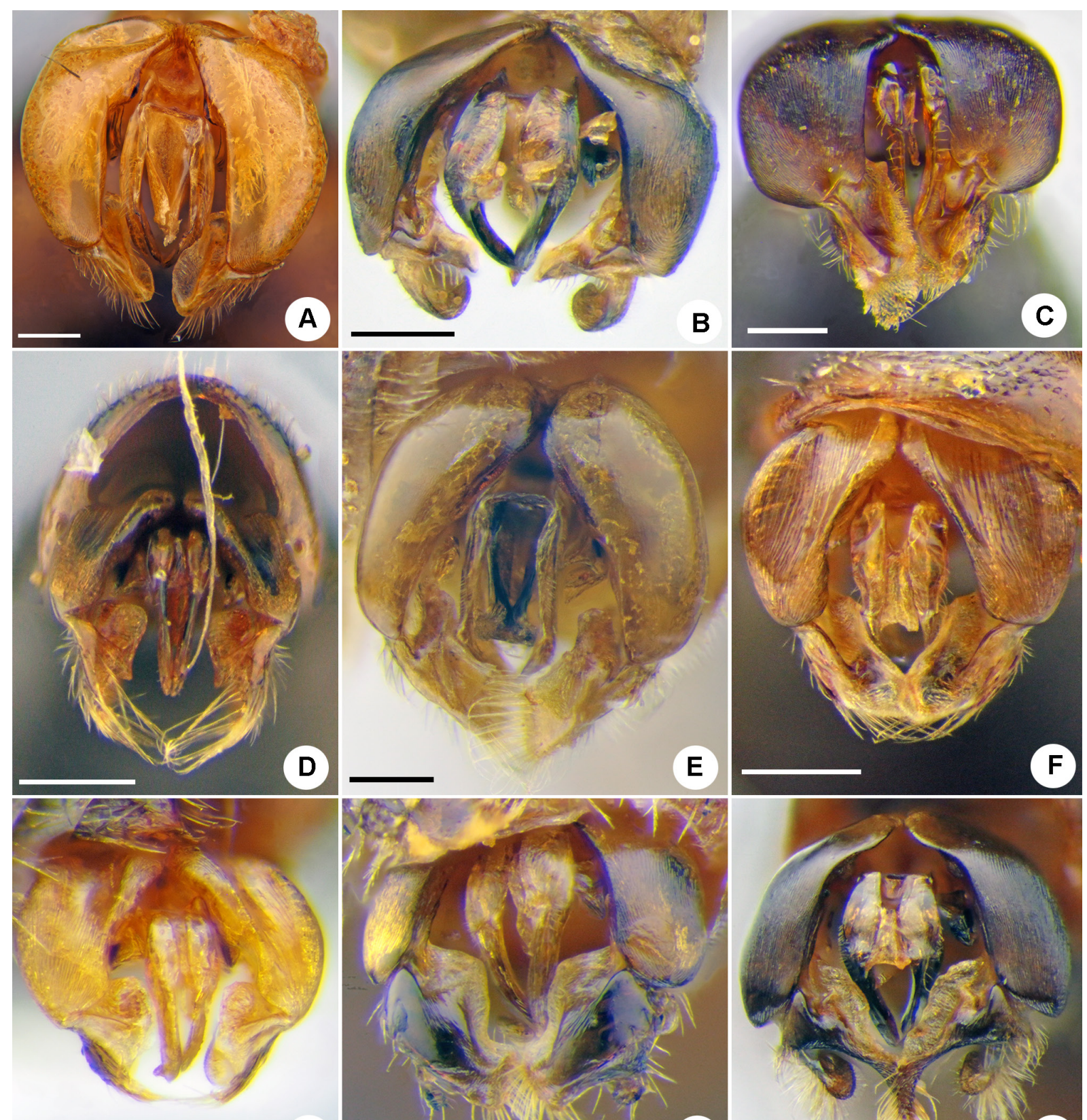

G
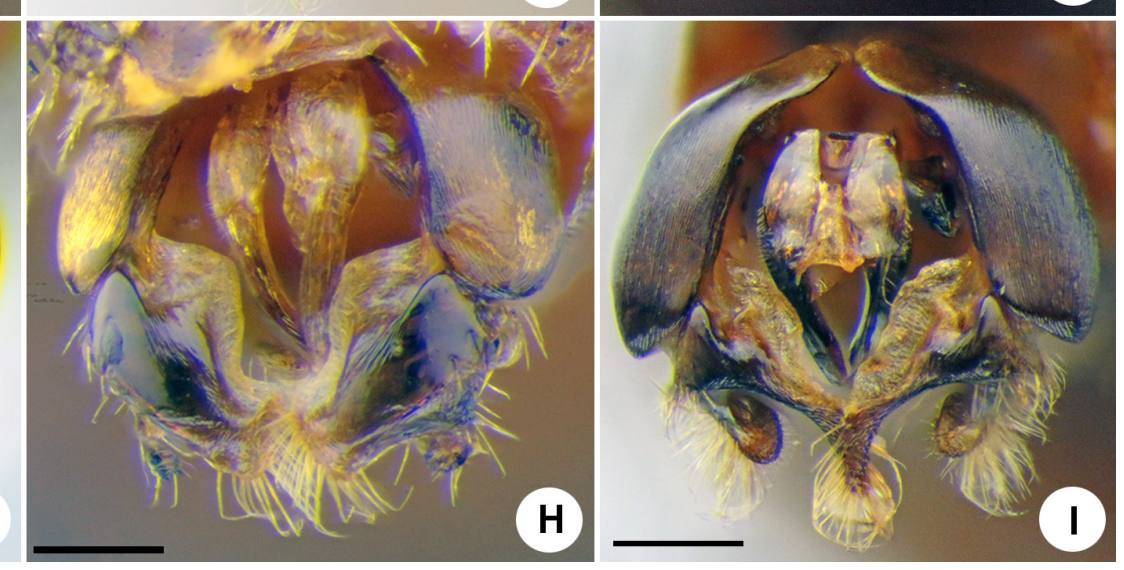

Fig. 3. Genitalia in dorsal view, males. A. Sphecodes fumipennis Smith, 1853. B. S. monilicornis (Kirby, 1802). C. S. montanus Smith, 1879. D. S. hakkariensis Warncke, 1922. E. S. albilabris (Fabricius, 1793). F. S. intermedius Blüthgen, 1923. G. S. simlaensis Blüthgen, 1924. H. S. rufiventris (Panzer, 1798). I. S. gibbus (Linnaeus, 1758). Scale bars $=0.25 \mathrm{~mm}$. 
11. Vertex long, distance from top of head to upper margin of lateral ocellus approximately three lateral ocellar diameters as seen in dorsal view. Tyloids usually well developed, covering at least entire lateral flagellar surface (with medial glabrous spot, variable in size, on ventral surface) .............. 12

- Vertex shorter, distance from top of head to upper margin of lateral ocellus ca two lateral ocellar diameters as seen in dorsal view. Tyloids weakly developed, covering basal $1 / 7-1 / 3$ of ventral flagellar surface

12. F3 long, ca 2 times as long as wide (Fig. 2E). Gonostylus elongate, with long apical process (Fig. 3I), apically less emarginated as seen in lateral view. Body length 7.0-14.0 mm

S. gibbus (Linnaeus, 1758)

- F3 short, 1.1-1.3 times as long as wide (Fig. 2F). Gonostylus without apical process (Fig. 3H), apically more emarginate as seen in lateral view. Body length $6.0-8.0 \mathrm{~mm}$

S. rufiventris (Panzer, 1798)

13. Tyloids less developed, covering basal $1 / 7-1 / 5$ of ventral flagellar surface (Fig. 2C). Body length $7.0-12.0 \mathrm{~mm}$ S. alternatus Smith, 1853

- Tyloids more developed, covering basal $1 / 5-1 / 3$ of ventral flagellar surface. Body length $10.0 \mathrm{~mm}$..

S. lasimensis Blüthgen, 1927

14. T1 densely punctate. Gonostylus large, rectangular and weakly $S$-curved on inner margin (Fig. 3F). Body length $5.0-7.5 \mathrm{~mm}$

S. intermedius Blüthgen, 1923

- T1 impunctate or with a few punctures. Gonostylus of another shape 15

15. Tyloids well developed, on flagellomeres (at least from F3 onward) covering entire ventral surface

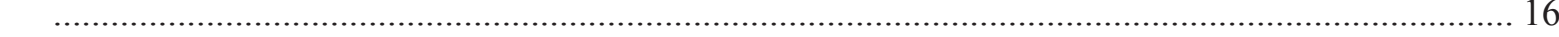

- Tyloids weakly developed, covering at most basal $1 / 4$ of ventral surface of last flagellomeres ...... 17

16. F2 with tyloids covering entire ventral surface (Fig. 2B). Hypoepimeral area entirely reticulaterugose. Base of gonocoxite dorsally without impression, gonostylus leaf-shaped. Body length 5.0$5.5 \mathrm{~mm}$ S. cameronii (Bingham, 1897)

- F2 glabrous, without tyloids (Fig. 2A). Hypoepimeral area medially smooth. Base of gonocoxite dorsally with impression, gonostylus trapezoidal (Fig. 3D). Body length 4.5-6.0 mm

S. hakkariensis Warncke, 1992

17. Vertex with longitudinal carina (Fig. 4B). Lateral preoccipital carina present (Fig. 4A). Gonostylus with large rectangular membranous part (Fig. 3C). Body length 7.0-7.5 mm

S. montanus Smith, 1879

- Vertex without longitudinal carina. Lateral preoccipital carina absent. Gonostylus smaller, of another shape

18. Antennae shorter, with flagellomeres (from F3 onward) ca 1.1-1.2 times as long as wide. Gonostylus with triangular membranous part (Fig. 3G). Body length 5.0-5.5 mm

S. simlaensis Blüthgen, 1924

- Antennae longer, with flagellomeres (from F3 onward) ca 1.3 times as long as wide. Gonostylus without membranous part. Body length $5.0 \mathrm{~mm}$ S. shillongensis Blüthgen, 1927

\section{Females}

1. Costal margin of hind wing with 7-14 hamuli. Usually larger species: total body length $6.5-$ $15.0 \mathrm{~mm}$

- Costal margin of hind wing with 5-6 hamuli. Usually smaller species: total body length 4.0$8.5 \mathrm{~mm}$ 

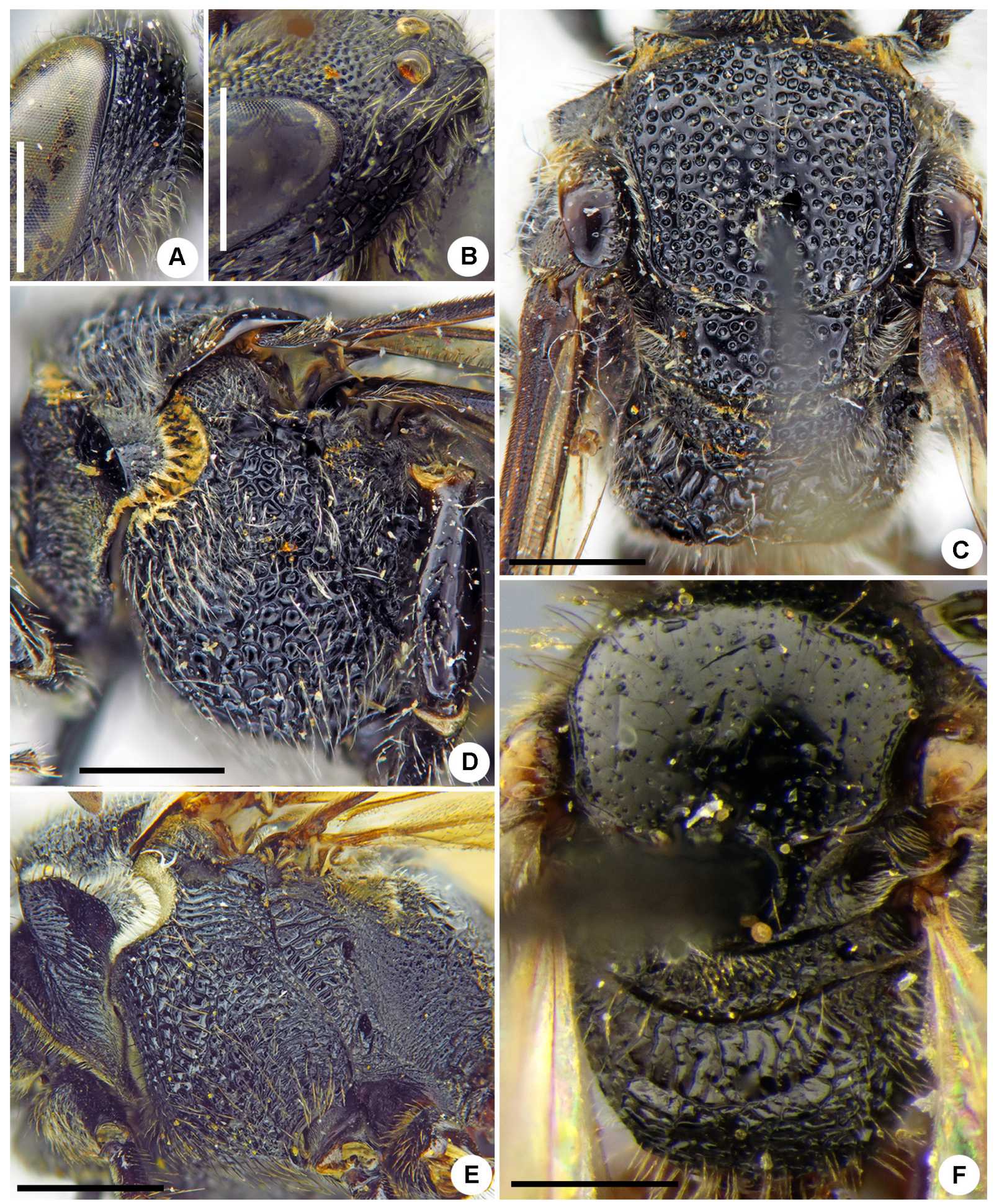

Fig. 4. Diagnostic characters of species of Sphecodes. A-B. Males. C-F. Females. A. Genal area and lateral preoccipital carina in lateral view. B. Vertex and longitudinal carina in dorso-lateral view. C-F. Mesosoma in dorsal (C, F) and lateral views (D-E). A-B. S. montanus Smith, 1879. C-D. S. fumipennis Smith, 1853. E. S. albilabris (Fabricius, 1793). F. S. hakkariensis Warncke, 1922. Scale bars $=1.0 \mathrm{~mm}$. 


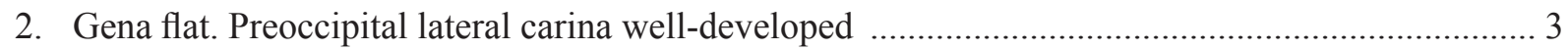

- Gena swollen. Preoccipital carina not developed …….................................................................... 7

3. Fore wing with two cubital cells. Body length $8.0-9.0 \mathrm{~mm}$....................S. turneri Cockerell, 1916

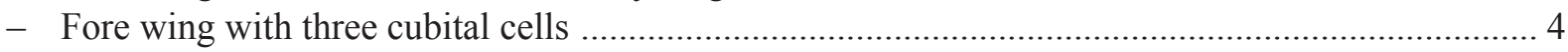

4. Large species: total body length $12.0-14.0 \mathrm{~mm}$. Costal margin of hind wing with $11-15$ hamuli .... S. sikkimensis Blüthgen, 1927

- Smaller species: total body length 7.0-9.0 mm. Costal margin of hind wing with 6-9 hamuli ....... 5

5. Head strongly transverse (Fig. 5B), ca 1.3 times as wide as long. T1 impunctate. Body length 7.0$8.0 \mathrm{~mm}$ S. laticeps Meyer, 1920

- Head less transverse (Fig. 5D), ca 1.2 times as wide as long. T1 distinctly and densely punctate .. 6

6. Mesoscutum with coarse and confluent punctures, but medially with interspaces $0.5-1$ puncture diameter. Costal margin of hind wing with $8-9$ hamuli. Pygidial plate as wide as metabasitarsus. Body length 8.0-9.0 mm

S. binghami Blüthgen, 1924

- Mesoscutum mostly areolate-punctate. Costal margin of hind wing with 6-7 hamuli. Pygidial plate 0.7 times as wide as metabasitarsus. Body length 7.5-8.0 $\mathrm{mm}$ S. kershawi Perkins, 1921

7. Mesoscutum densely punctate, with punctures separated by less than a puncture diameter (Fig. 4C)

- Mesoscutum sparsely punctate, medially with some punctures separated by at least two puncture diameters (Fig. 4F) ....

8. Paraocular areas with dense plumose pubescence, obscuring integument. Mesepisternum reticulaterugose (Fig. 4E). T1 densely punctate, with punctures separated by $0.5-2$ puncture diameters. Body length $9.0-15.0 \mathrm{~mm}$

S. albilabris (Fabricius, 1793)

- Paraocular areas with relatively dense plumose pubescence, not obscuring integument. Mesepisternum areolate (Fig. 4D). T1 sparsely punctate, with punctures separated by 2-6 puncture diameters. Body length 9.5-12.0 mm

S. fumipennis Smith, 1853

9. Terga with marginal zones almost impunctate, on T1 only with a few tiny punctures. Body length $7.5-10.0 \mathrm{~mm}$ S. lasimensis Blüthgen, 1927

- Terga with marginal zones distinctly punctate at least on T1 and T2 10

10. Vertex shorter, distance from top of head to upper margin of lateral ocellus ca two lateral ocellar diameters as seen in dorsal view. Propodeum laterally reticulate-rugose. T3 with marginal zone distinctly punctate. Body length $8.0-11.0 \mathrm{~mm}$

S. alternatus Smith, 1853

- Vertex longer, distance from top of head to upper margin of lateral ocellus equal to 2.5-3.0 lateral ocellar diameters as seen in dorsal view. Propodeum laterally strigate or reticulate-rugose. T3 with marginal zone impunctate or punctate

11. Head rounded-rectangular on upper margin, square-shaped as seen in frontal view (Fig. 5C); vertex sparsely punctate, punctures mostly separated by more than a puncture diameter. Hind wing with basal vein strongly curved, the angle between basal $(M)$ and cubital $(\mathrm{Cu})$ veins ca $90^{\circ}$. T1 indistinctly punctate, with a few fine punctures. Pygidial plate 0.9-1.0 times as wide metabasitarsus. Body length $7.0-10.0 \mathrm{~mm}$

S. monilicornis (Kirby, 1802)

- Head uniformly rounded on upper margin, oval as seen in frontal view; vertex densely punctate, punctures mostly separated by less than a puncture diameter. Hind wing with basal vein weakly 
curved, the angle between basal $(M)$ and cubital $(\mathrm{Cu})$ veins ca $70-80^{\circ}$. T1 distinctly punctate, with fine and coarser punctures. Pygidial plate $0.5-0.6$ times as wide as metabasitarsus

12. Mesepisternum strigate or strigate-rugose at least ventrally, sides of propodeum strigate. T3 with marginal zone coarsely punctate. Body length $6.5-8.5 \mathrm{~mm}$ S. rufiventris (Panzer, 1798)

- Mesepisternum reticulate-rugose, sides of propodeum strigate or reticulate-rugose. T3 with marginal zone impunctate. Body length 7.0-15.0 mm S. gibbus (Linnaeus, 1758)

13. Mandible simple (without an inner tooth) 14

- Mandible bi-dentate

14. Paraocular and supraclypeal areas with moderately dense pubescence, not obscuring integument. F1 and F2 as long as wide. Mesoscutum with punctures separated by at most a puncture diameter.
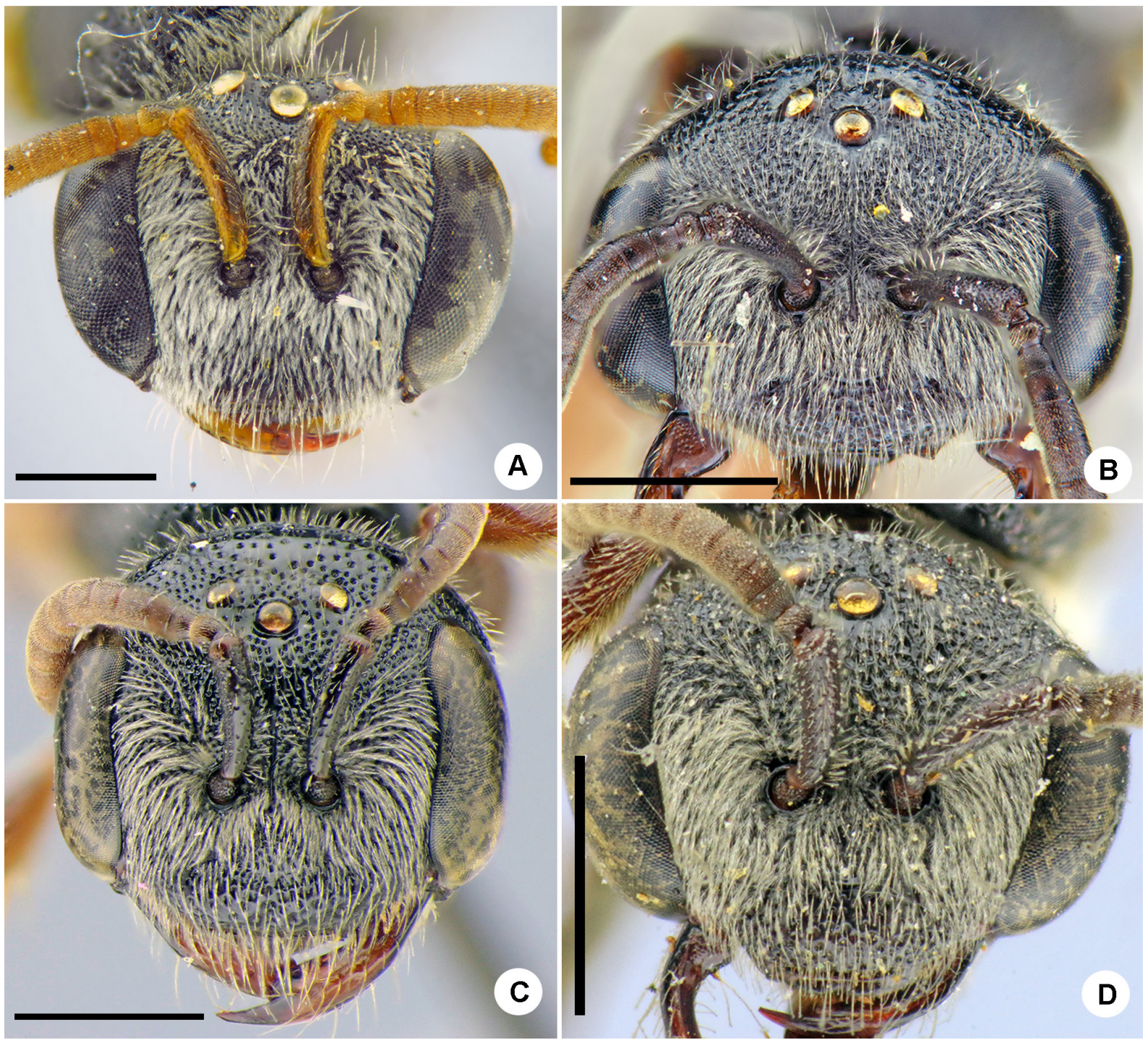

Fig. 5. Head in frontal view, females. A. Sphecodes cameronii (Bingham, 1897). B. S. laticeps Meyer, 1920. C. S. monilicornis (Kirby, 1802). D. S. kershawi Perkins, 1921. Scale bars $=1.0 \mathrm{~mm}$. 
Metasomal terga (except almost impunctate T1) coarser punctate $(10-15 \mu \mathrm{m})$. Pygidial plate as wide as metabasitarsus. Body length $5.5 \mathrm{~mm}$

S. setiger Blüthgen, 1924

- Paraocular and supraclypeal areas with dense pubescence, obscuring integument (Fig. 5A). F1 and F2 0.6-0.7 times as long as wide. Mesoscutum finely and irregularly punctate with punctures separated by $1-5$ puncture diameters. Metasomal terga finely punctate $(3-10 \mu \mathrm{m})$. Pygidial plate 1.4-1.5 times as wide as metabasitarsus. Body length 5.0-5.5 mm

S. cameronii (Bingham, 1897)

15. Vertex with longitudinal carina. Lateral preoccipital carina present, but weakly developed. Pygidial plate wider, 1.1-1.2 times as wide as metabasitarsus. Body length 7.0-7.5 mm

S. montanus Smith, 1879

- Vertex without longitudinal carina. Lateral preoccipital absent. Pygidial plate narrower, at most as wide as metabasitarsus

16. Pronotum rounded between the dorsal and lateral surfaces. Body length 7.0-7.5 mm (Fig. 6)

S. bluethgeni sp. nov.

- Pronotum between dorsal and lateral surfaces with sharp angle

17. T1 distinctly punctate. Pygidial plate narrow, 0.4 times as wide as metabasitarsus. Body length $6.5-8.5 \mathrm{~mm}$

S. intermedius Blüthgen, 1923

- T1 impunctate or with sparse tiny punctures. Pygidial plate wider, at least 0.7 times as wide as metabasitarsus

18. Head strongly transverse, ca 1.3 times as wide as long. Metasomal terga entirely red

- Head less transverse, ca 1.2 times as wide as long. T4 and T5 at least partially brownish or black ..

19. Smaller species: total body length $4.0 \mathrm{~mm}$. Legs entirely black

S. almoransis Gupta \& Saini, 2018

- Larger species: total body length $6.5-8.0 \mathrm{~mm}$. Legs entirely red or at least tarsi red or yellow .... 20

20. Mesoscutum denser punctate, with punctures separated by 1-2 puncture diameters. Pygidial plate as wide as metabasitarsus. Body length $6.5 \mathrm{~mm}$

S. perplexus Nurse, 1903

- Mesoscutum sparser punctate, with punctures separated by 2-5 puncture diameters. Pygidial plate 0.8 times as wide as metabasitarsus. Body length $8.0 \mathrm{~mm}$ S. tantalus Nurse, 1903

21. Mesoscutum sparsely punctate, with punctures separated by 2-6 puncture diameters (Fig. 4F). Hypoepimeral area medially smooth. Body length 4.5-5.0 mm ......S. hakkariensis Warncke, 1992

- Mesoscutum denser punctate, with punctures separated by 1-4 puncture diameters. Hypoepimeral area entirely rugose

22. Head less transverse, 1.1 times as wide as long. Legs entirely red. Body length $4.5 \mathrm{~mm}$

S. iridipennis Smith, 1879

- Head more transverse, 1.2 times as wide as long. At least femora black or brownish. Body length $5.0-6.5 \mathrm{~mm}$ S. simlaensis Blüthgen, 1924 


\section{Himalayan species}

Class Insecta Linnaeus, 1758

Order Hymenoptera Linnaeus, 1758

Family Halictidae Thomson, 1869

Tribe Halictini Thomson, 1869

Genus Sphecodes Latreille, 1804

Sphecodes albilabris (Fabricius, 1793)

Figs 3E, 4E

Nomada albilabris Fabricius, 1793: 349, ㅇ, ô (type locality: Germany).

Dichroa fuscipennis Germar, 1819: 18, $q$ (type locality: Germany).

Sphecodes latreillei Wesmael, 1835: 285, , Ő (type locality: Belgium).

Sphecodes nigripes Lepeletier de Saint Fargeau, 1841: 542,, , $\widehat{O}$ (type locality: France).

Sphecodes rugosus Smith, 1848: 2209, đ̊ (type locality: England).

Sphecodes nodicornis Gistel, 1857: 554, ㅇ, §ิ (type locality: Germany).

Sphecodes fuscipennis var. basalis Dalla Torre, 1877 (nom. praeocc., nec Sichel 1865): 185, o (type locality: Italy).

Sabulicola cirsii Verhoeff, 1890: 329, ô (type locality: Germany).

Sphecodes grandis Meyer, 1922: 173, ô (type locality: Northeast India).

Sphecodes rufipennis Cockerell, 1931a: 348, ô (type locality: Morocco).

Sphecodes atrescens Cockerell, 1931a: 350, §̂ (type locality: Morocco).

Sphecodes albilabris - Astafurova \& Proshchalykin 2017b: 251.

\section{Diagnosis and descriptive notes}

See Astafurova et al. (2018a: 5).

\section{Material examined}

INDIA • 1 §̊; Himachal Pradesh, Tabo; 2800 m a.s.1.; 11 Jul. 2001; V. Major leg.; PCMS.

NEPAL • 1 q; Mustang Distr., Purano Marpha; 3200 m a.s.1.; 9-11 May 1995; Iglesias leg.; PCMS.

\section{Distribution}

Himalayas: Tajikistan (Gorno-Badakhshan Province), India (*Himachal Pradesh), *Nepal; North Africa, Europe (north to Finland and Sweden), Russia (east to Primorsky Terr.), Turkey, Caucasus, Kazakhstan, Central Asia, Iran, North and North East China, India (Uttar Pradesh).

\section{Sphecodes binghami Blüthgen, 1924}

Sphecodes binghami Blüthgen, 1924: 497, q.

\section{Diagnosis and descriptive notes}

See Astafurova et al. (2020b: 37).

\section{Material examined}

Holotype

MYANMAR • +; Pegu Hills; Mar. 1889; Bingham leg.; NHMUK 010576231. 


\section{Other material}

NEPAL • 1 क ; Kali-Gandaki-Kola, Tatopani, 50 km SW of Pokhara; $28^{\circ} 20^{\prime}$ N, 8330' E; 1100-1200 m a.s.1.; C. Holzschuh leg.; PCMS.

\section{Distribution}

Himalayas: *Nepal; Myanmar, Malaysia.

Sphecodes bluethgeni sp. nov.

urn:1sid:zoobank.org:act:E7F25913-AF79-4CC7-8E33-B6470F31813F

Figs 6, 7A-D

\section{Diagnosis}

Like members of the Palaearctic hyalinatus species group (see Astafurova \& Proshchalykin 2017a) the new species has a pronotum rounded between the dorsal and lateral surfaces, but it clearly differs by the presence of a sharp carina between the lateral and vertical surfaces of the propodeum (Fig. 7D). From S. engeli Astafurova \& Proshchalykin, 2020, another Oriental species of this group, the new species differs by the shape of the head with a rounded vertex as seen in frontal view (vs the head with a straight upper margin in S. engeli).

\section{Etymology}

This species is dedicated to famous German entomologist Paul Blüthgen (1880-1967), in recognition of his significant contributions to the study of bees and wasps.

\section{Material examined}

\section{Holotype}

BHUTAN • +; Paro Prov., Chiley La; 3000-3500 m a.s.1.; 10-13 Jul. 1990; C. Holzschuh leg.; PCMS/ OLBL.

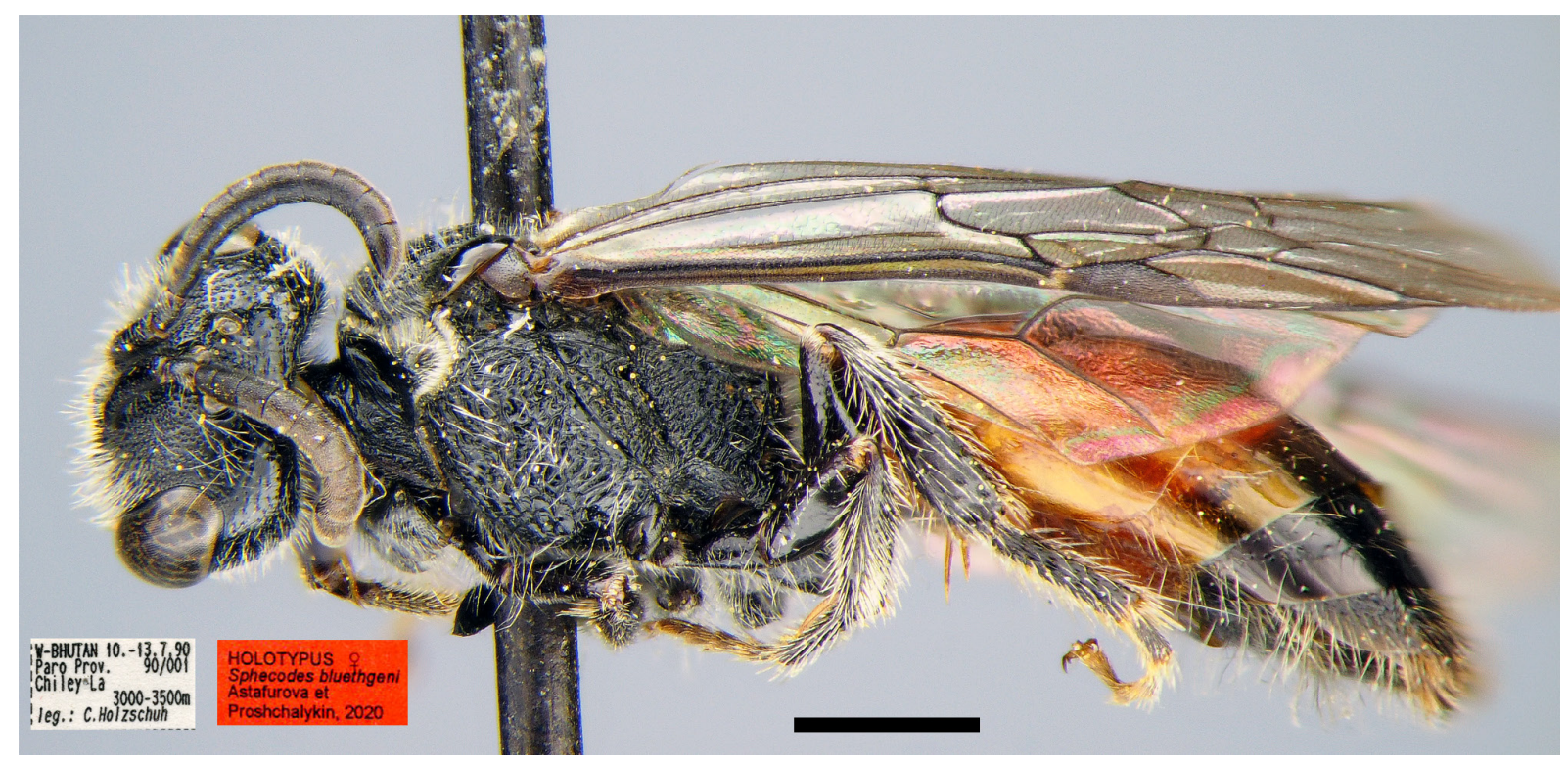

Fig. 6. Sphecodes bluethgeni sp. nov. Holotype, $q$ (PCMS/OLBL). Habitus in lateral view and labels. Scale bar $=1 \mathrm{~mm}$. 

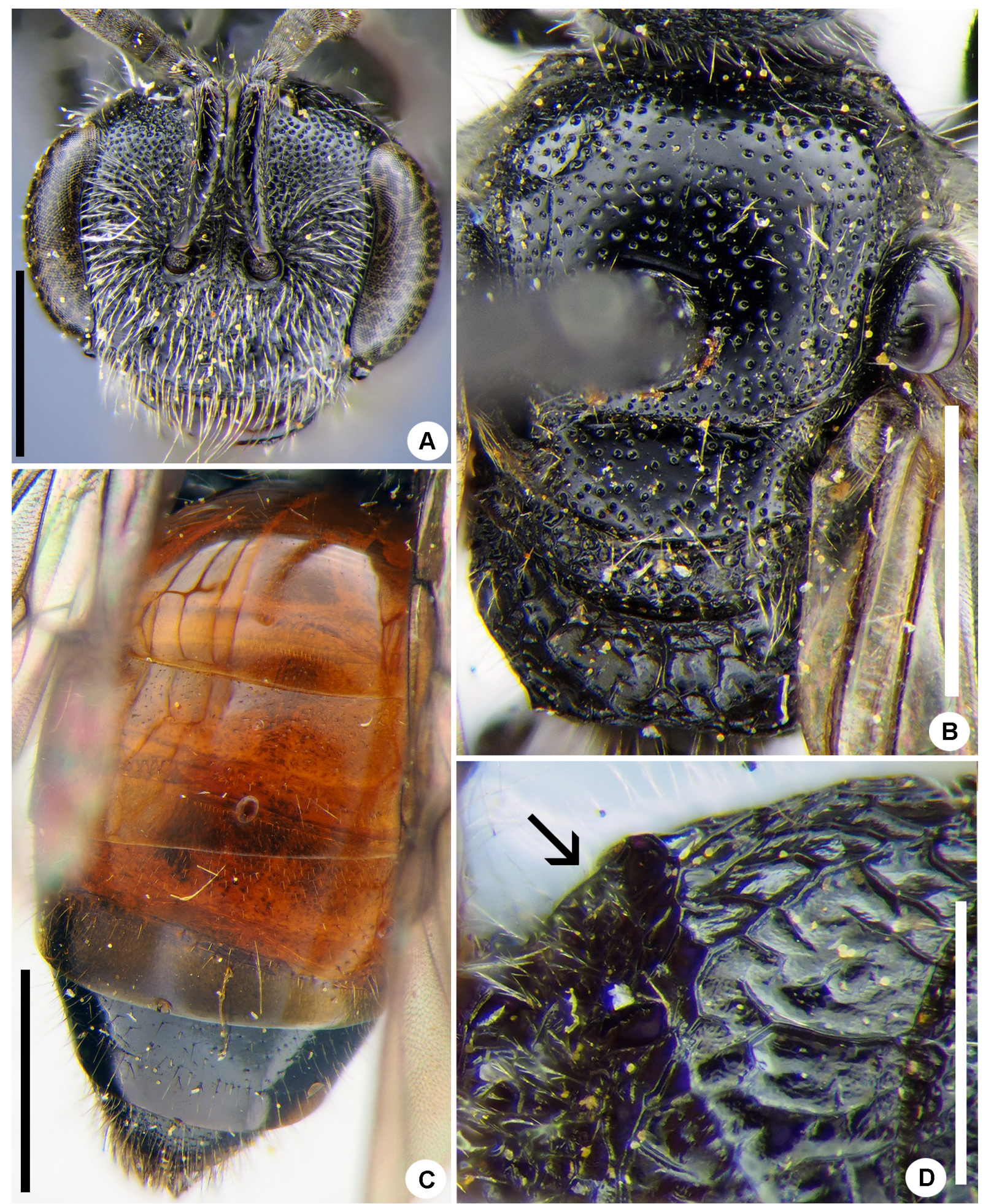

Fig. 7. Sphecodes bluethgeni sp. nov. Holotype, $q$ (PCMS/OLBL). A. Head in frontal view. B. Mesosoma in dorsal view. C. Metasoma in dorsal view. D. Propodeum in dorso-lateral view. Scale bars $=1 \mathrm{~mm}$. 


\section{Paratype}

BHUTAN • 1 q; Thimpu Distr., Taba; 2600 m a.s.1;; 14-17 Jul. 1988; C. Holzschuh leg.; ZISP.

\section{Description}

Female (Fig. 6, holotype)

MEASUREMENTS. Total body length $7.0-7.5 \mathrm{~mm}$; forewing length (without tegula) $5.8-6.0 \mathrm{~mm}$.

HEAD. Black (Fig. 7A); transverse, ca 1.25 times as wide as long; preoccipital carina absent; vertex weakly elevated, distance from top of head to upper margin of a lateral ocellus approximately a lateral ocellar diameter as seen in frontal view and ca 2.5 diameters as seen in dorsal view; mandible bi-dentate; labrum semi-oval, 0.5 times as long as basal width; F1 0.7 times as long as wide; F2 0.8-0.9 times as long as wide; F3 0.9-1.0 times as long as wide; gena wide, 1.1 times as wide as eye; supraclypeal area weakly bulging; clypeus with punctures separated by at most a half puncture diameter; frons, paraocular and supraclypeal areas with confluent punctures $(20-25 \mu \mathrm{m})$; ocello-ocular area with punctures separated by 0.5-2 puncture diameters (Fig. 7A), vertex behind ocelli shiny with sparse punctures; gena shiny, with dense setal pores; face (below antennal sockets) and gena with sparse and thin setae.

Mesosoma. Mesosoma (including legs) black (Fig. 7B); wings with weak brownish darkening, veins and stigma brown; hind wing with angle between basal $(M)$ and cubital $(C u)$ veins ca $90^{\circ}$, costal margin with six hamuli; pronotum rounded between dorsal and lateral surfaces; mesoscutum and mesoscutellum with punctures $(25-30 \mu \mathrm{m})$ separated by $0.5-4$ puncture diameters; metafemur enlarged in proximal half, maximum width 0.4 times its length; hypoepimeral area, mesepisternum and lateral parts of propodeum reticulate-rugose; propodeal triangle (metapostnotum) with coarse winding wrinkles and large shiny, finely granulate interspaces (Fig. 7B, D).

Metasoma. T1 almost impunctate, with a few fine punctures along marginal zone (Fig. 7C); remaining terga basally with sparse and fine punctures $(5-10 \mu \mathrm{m})$; marginal zones impunctate; pygidial plate dull, pointed apically, narrow, $0.8-0.9$ times as wide as metabasitarsus; T1-T3 red; sterna tessellate, with tiny shallow setal pores (sparse on S2 and denser on S3-S5).

Male

Unknown.

\section{Distribution}

Bhutan.

Sphecodes cameronii (Bingham, 1897)

Figs 2B, 5A

Halictus decorus Cameron, 1897: 94, q (nom. praeocc., nec Halictus decorus Walker, 1871).

Halictus cameronii Bingham, 1897: 432, replacement name for H. decorus Cameron, 1897 (nec H. decorus Walker, 1871).

Sphecodes armeniacus Warncke, 1992: 19, ㅇ, §̊. Syn. nov.

Sphecodes decorus - Cameron 1897: 94. — Gupta 2013: 60.

Sphecodes cameronii - Cockerell 1921: 360. — Ascher \& Pickering 2020: map.

\section{Diagnosis and descriptive notes}

See Astafurova et al. (2018a: 9, as S. armeniacus Warncke, 1992). 


\section{Material examined}

INDIA • 1 \%, syntype of Halictus decorus Cameron, 1897; "Mussouri [Mussoorie, Uttarakhand, India] // Sphecodes // 999. // Halictus decorus Cameron, Type // Type Hyme 1956, Halictus decorus Cameron,

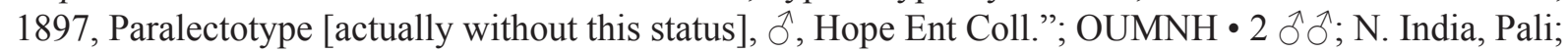
5 Mar. 1997; K.M. Guichard leg.; NHMUK 013380471, NHMUK 013380451.

NEPAL • 1 क; Rapti Tal, Monahari Khola, Belwa; 350 m a.s.1.; 10 May 1967; Dierl-Forster-Schacht leg.; PCMS.

TURKEY 1 1 , holotype of Sphecodes armeniacus; Kars, Tuzluca; OLBL/PCMS.

\section{Distribution}

Himalayas: *Nepal, India (Uttarakhand); Turkey, South Kazakhstan, Turkmenistan, India (Pali), Sri Lanka (Inoka et al. 2005).

\section{Sphecodes dissimilandus (Cameron, 1897)}

Fig. 8A-D

Halictus dissimilandus Cameron, 1897: 95, 우 [ô]].

Sphecodes dissimulandus - Blüthgen 1927: 90 (incorrect spelling).

Sphecodes dissimilandus - Ascher \& Pickering 2020: map.

\section{Diagnosis}

The male of this species is similar to $S$. invidus owing to a similar shape of antennal tyloids (well developed, covering entire lateral flagellar surface and a part of ventral one), sculpture and coloration of the body, including hyaline wings, the areolate mesoscutum, the densely punctate T1 on the disc and the marginal zone. The species clearly differs from $S$. invidus by the weaker elevated vertex as seen in frontal view, with distance from top of head to upper margin of lateral ocellus ca 1.5 lateral ocellar diameter (vs 2 in S. invidus) and the finer punctate metasoma (10-20 $\mu \mathrm{m}$ vs $15-25 \mu \mathrm{m})$.

\section{Material examined}

\section{Holotype}

INDIA • J; "Mussouri 74[Mussoorie, Uttarakhand, India], Rothney [leg.]"; "Sphecodes // 998. // Halictus dissimilandus Cameron, Type // Type Hyme 1957, Halictus dissimilandus Cameron, 1897, Holotype, ô, Hope Ent Coll.”; OUMNH.

\section{Descriptive notes}

Wings hyaline, without darkening; hind wing with the angle between basal $(M)$ and cubital $(\mathrm{Cu})$ veins ca $80^{\circ}$, costal margin with seven hamuli. Lateral preoccipital carina present.

\section{Male}

Total body length $7 \mathrm{~mm}$. Head transverse, ca 1.2 times as wide as long (Fig. 8D); vertex elevated with distance from top of head to upper margin of lateral ocellus ca 1.5 lateral ocellar diameter as seen in frontal view and ca 2 as seen in dorsal view (Fig. 8A); antennae attain mesoscutellum, flagellomeres (from F2 onward) 1.2-1.3 times as long as wide, tyloids well developed, covering entire lateral flagellar surface and most part (from F4 onward) of ventral one (with small medial glabrous spot); ocello-ocular area close to areolate, with dense punctures separated by at most a half puncture diameter; face with dense plumose pubescence, below antennal sockets obscuring integument. Mesoscutum areolate- 
punctate $(30-50 \mu \mathrm{m})$, mesoscutellum with interspaces equal to at most 2 puncture diameters (Fig. 8A); propodeal triangle (metapostnotum) shorter than mesoscutellum, roughly rugose; mesepisternum coarsely reticulate-rugose; legs red. Metasomal terga finely punctate (10-20 $\mu \mathrm{m} / 1-4)$, T1 marginal zone punctured basally, T2-T5 marginal zones impunctate; metasoma entirely red (Fig. 8B).

\section{Female}

Unknown.

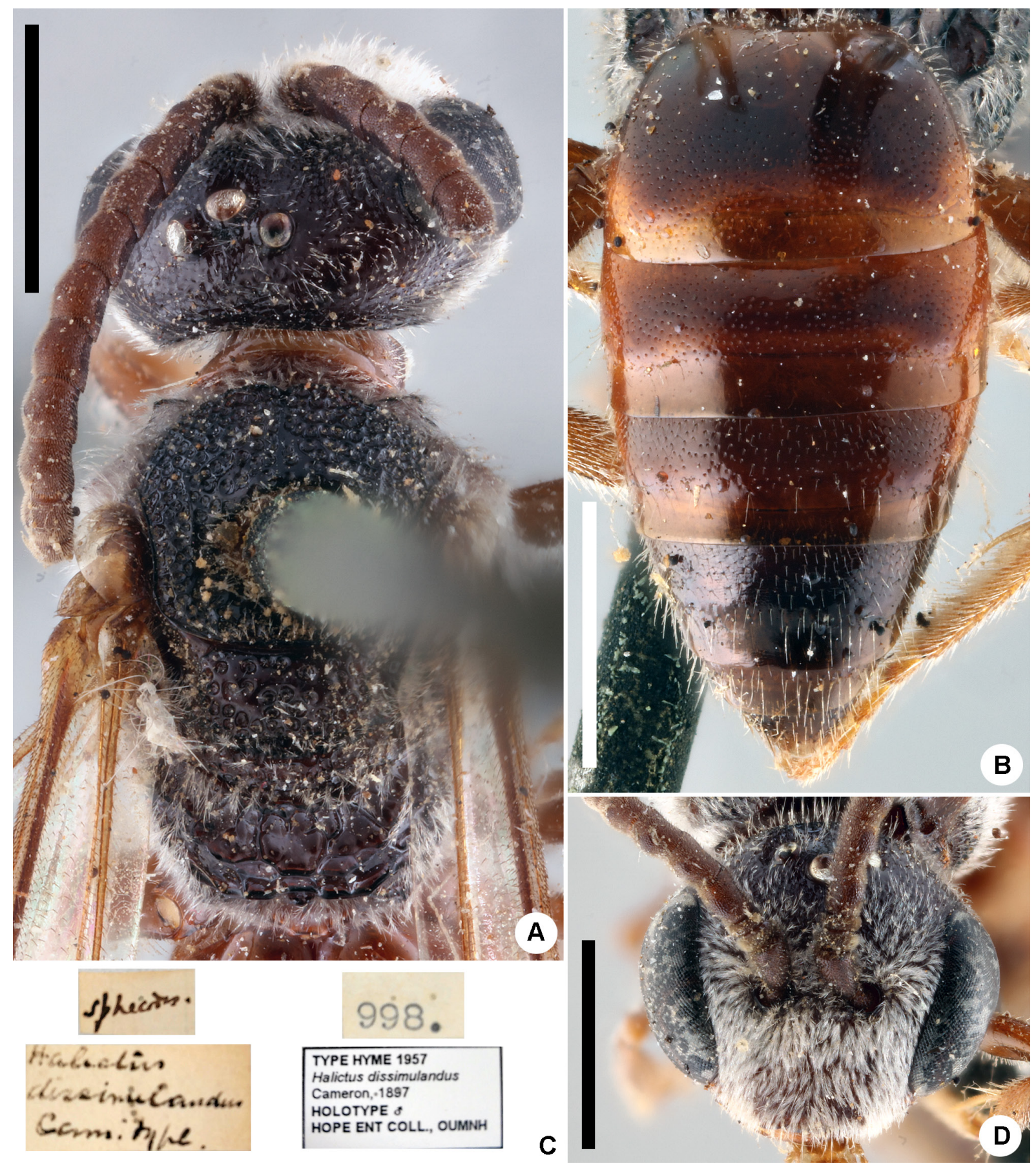

Fig. 8. Sphecodes dissimilandus (Cameron, 1897). Holotype, ô (OUMNH). A. Head and mesosoma in dorsal view. B. Metasoma in dorsal view. C. Labels. D. Head in frontal view. Scale bars $=1 \mathrm{~mm}$. 


\section{Distribution}

Himalayas: India (Uttarakhand).

Sphecodes fumipennis Smith, 1853

Figs 3A, 4C-D

Sphecodes fumipennis Smith, 1853: 36, ㅇ (holotype: + , N. India, coll. J.S. Baly; London, NHMUK; not examined).

Sphecodes fumipennis - Blüthgen 1924: 489; 1927: 55. — Ascher \& Pickering 2020: map.

\section{Diagnosis and descriptive notes}

See Astafurova et al. (2020b: 55).

\section{Material examined}

INDIA • 1 q, 1 đ̊; Sikkim; Binghan leg. [Sph. fumipennis Smith, Blüthgen det.]; ZMHB.

\section{Distribution}

Himalayas: India (Sikkim); Myanmar, Laos, China (Sichuan, Yunnan).

Sphecodes gibbus (Linnaeus, 1758)

Figs 2E, 3I

Sphex gibba Linnaeus, 1758: 571, o (type locality: Sweden).

Apis glabra Füessly, 1775: 51, o (type locality: Switzerland).

Andrena ferruginea Olivier, 1789: 139, nom. nov. for Nomada gibba Fabricius, 1775 (nom. praeocc., nec Linnaeus, 1758).

Apis gibbosa Christ, 1791: 177, nom. nov. for Nomada gibba Fabricius, 1775 (nom. praeocc., nec Linnaeus, 1758).

Melitta sphecoides Kirby, 1802: 46, \& (type locality: England).

Melitta picea Kirby, 1802: 48, ふ̊ (type locality: England).

Andrena austriaca Fabricius, 1804: 325, + (type locality: Denmark) (nom. praeocc., nec Panzer, 1798).

Dichroa analis Illiger, 1806: 48, nomen novum for Nomada gibba Fabricius, 1775 (nom. praeocc., nec Linnaeus, 1758).

Sphecodes apicatus Smith, 1853: 36, + (syntypes: + , India, Simla; NHMUK). Synonymized by Blüthgen 1927: 27.

Sphecodes nigripennis Morawitz, 1876: 257, $q$ (type locality: Kazakhstan).

Sphecodes indicus Bingham, 1898: 123, pl. a, fig. 10, đ̊. Syn. nov.

Sphecodes sutor Nurse, 1903: 538, ㅇ (syntypes: 우, India, Jammu and Kashmir; NHMUK). Synonymized by Blüthgen 1927: 27.

Sphecodes gibbus var. rufispinosus Meyer, 1920: 113, o (type locality: Spain).

Sphecodes gibbus var. turkestanicus Meyer, 1920: 113, +, О (type locality: Uzbekistan, "Golodnaja Steppe"; China: Jarkand, Xinjiang).

Sphecodes castilianus Blüthgen, 1924: 473, o (type locality: Portugal).

Sphecodes lustrans Cockerell, 1931b: 411, ô (type locality: Morocco).

Sphecodes angarensis Cockerell, 1937: 3-4, o (type locality: Russia, Irkutsk Prov.).

Sphecodes pergibbus Blüthgen, 1938: 50, ㅇ, ô (type locality: Cyprus). 
Sphecodes indicus - Saini \& Rathor 2012: 162. — Rajkumar \& Dey 2016: 1841.

Sphecodes gibbus - Ascher \& Pickering 2020: map.

\section{Diagnosis and descriptive notes}

See Astafurova et al. (2018a: 17).

\section{Material examined}

INDIA • 1 đ, holotype of Sphecodes indicus Bingham, 1898; "Type // Simla, 5. 97. // Sphecodes indicus (Bingh) // B.M.Type HYM 17a560"; NHMUK 013380329 • 1 \&; Simla; May 1898; C.G. Nurse leg.; NHMUK 013380335.

\section{Distribution}

Himalayas: India (Jammu and Kashmir, Himachal Pradesh); North Africa, Europe (north to $63^{\circ}$ latitude), Russia (east to Yakutia), Turkey, Israel, Jordan, Caucasus, Kazakhstan, Central Asia, Iran, Mongolia, China (Xinjiang).

\section{Remarks}

The record of S. indicus from Mussoorie (Uttarakhand, India) by Saini \& Rathor (2012: 162) is doubtful.

\section{Sphecodes invidus (Cameron, 1897)}

Halictus invidus Cameron, 1897: 96, ㅇ [ð].

Sphecodes nigrobasalis Meyer, 1922: 172, đ̂. Syn. nov.

Sphecodes invidus - Ascher \& Pickering 2020: map.

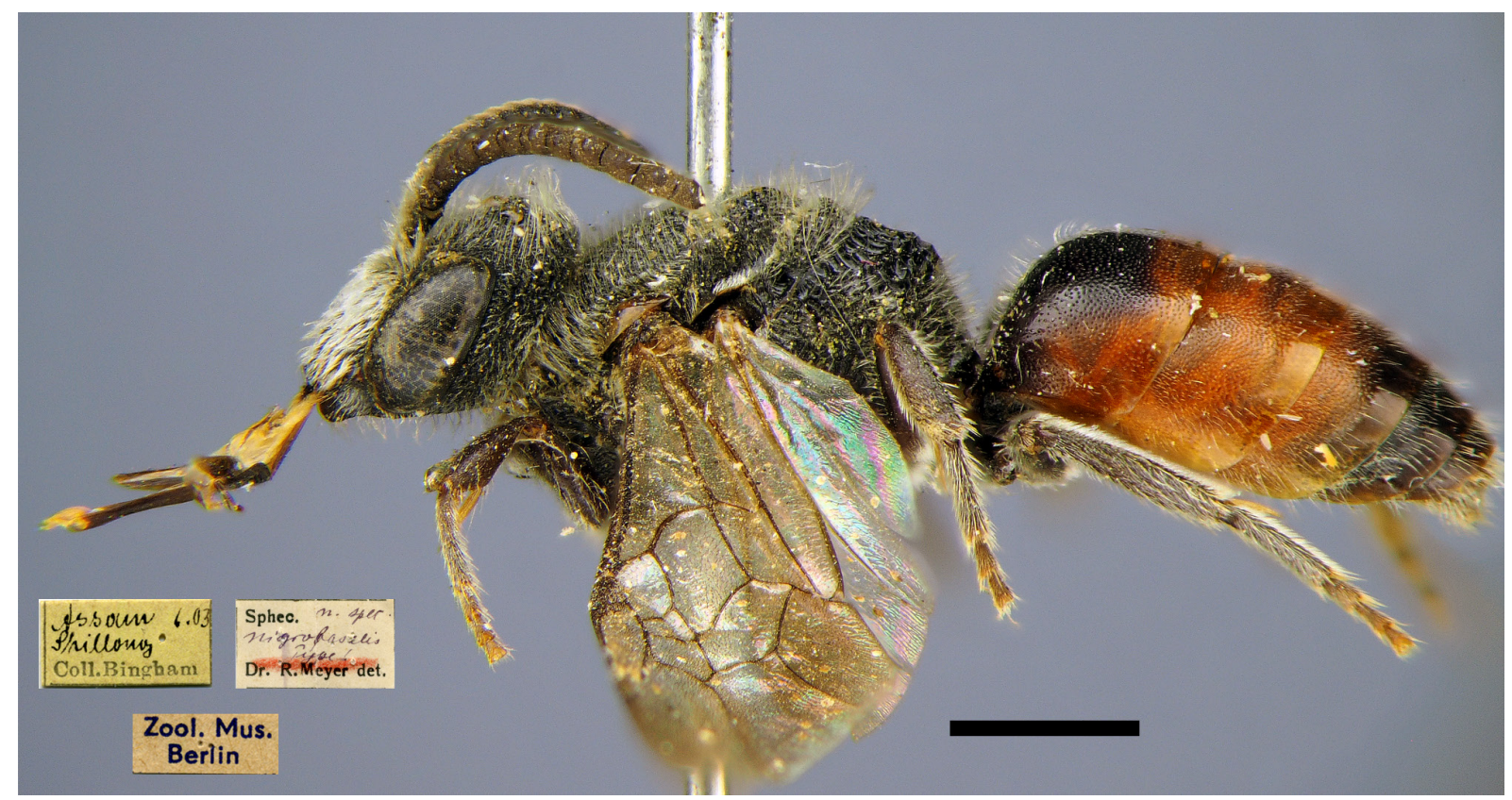

Fig. 9. Sphecodes invidus (Cameron, 1897) (holotype of S. nigrobasalis Meyer, 1922), § (ZMHB). Habitus in lateral view and labels. Scale bar $=1 \mathrm{~mm}$. 


\section{Diagnosis}

The male of this species is similar to that of $S$. dissimilandus (refer to Diagnosis for S. dissimilandus, above).

\section{Material examined}

\section{Syntype}

INDIA • 1 §; "1000. // Mussoorie [Uttarakhand, India], Rothney [leg.] // Halictus invidus Cameron, Type // Type Hyme 1958, Halictus invidus Cameron, 1897, Holotype, đ̊, Hope Ent Coll.”; OUMNH.
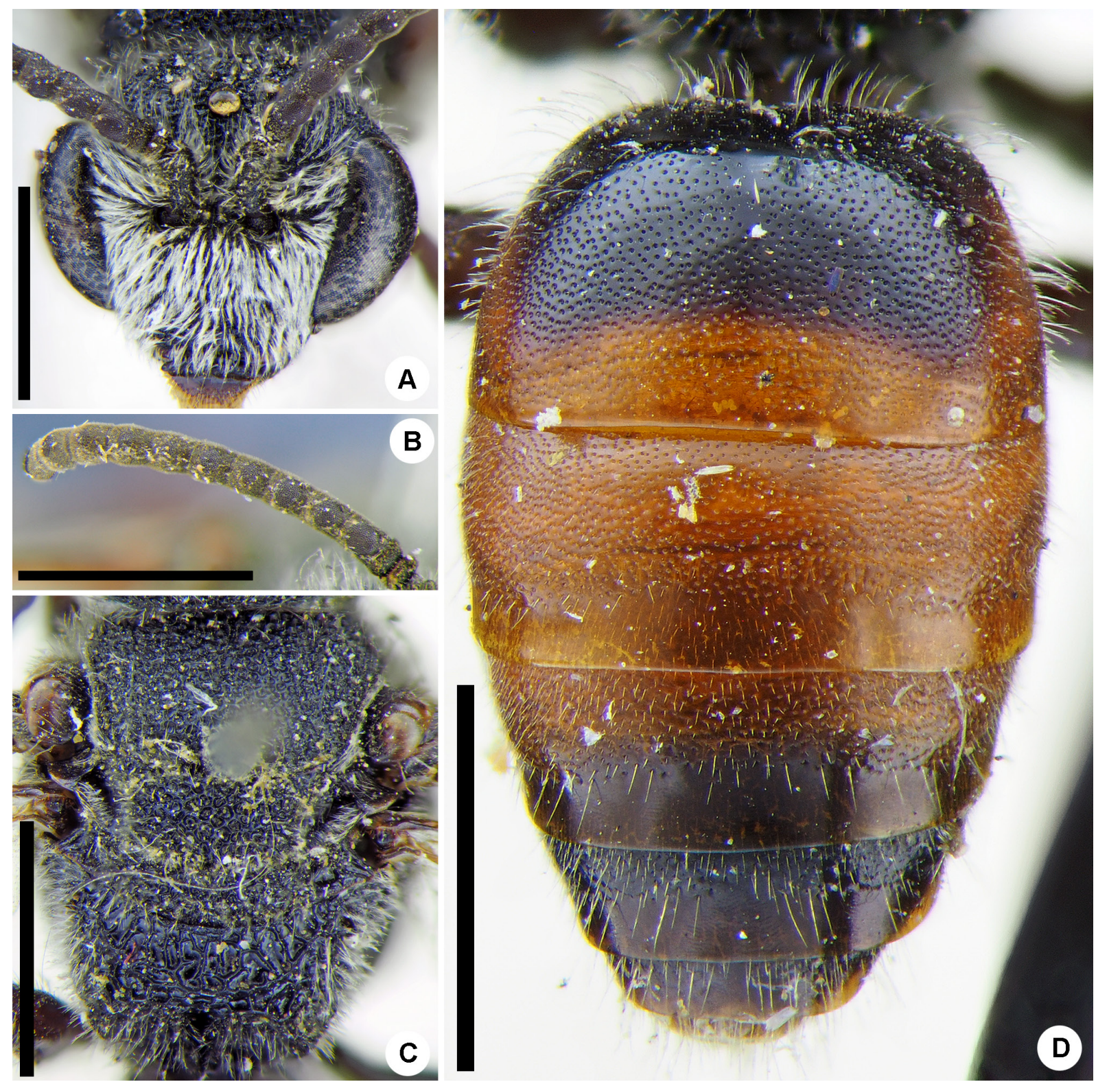

Fig. 10. Sphecodes invidus (Cameron, 1897) (holotype of S. nigrobasalis Meyer, 1922), ô (ZMHB). A. Head in frontal view. B. Antenna in lateral view. C. Mesosoma in dorsal view. D. Metasoma in dorsal view. Scale bars $=1.0 \mathrm{~mm}$. 


\section{Other material}

INDIA 1 $\widehat{\partial}$, holotype of S. nigrobasalis Meyer, 1922; “Assam [India], Shillong 6.03., coll. Bingham // Sphec. nigrobasalis n. scec., Dr. R. Meyer det., Type!”; ZMHB (Figs 9, 10A-D).

\section{Descriptive notes}

Wings hyaline, with weak yellowish darkening; hind wing with the angle between basal $(M)$ and cubital $(\mathrm{Cu})$ veins ca $80^{\circ}$, costal margin with eight or nine hamuli. Lateral preoccipital carina present.

\section{Male}

Total body length 7.5-8.5 mm. Head transverse, ca 1.2 times as wide as long; vertex well elevated with distance from top of head to upper margin of lateral ocellus approximately two lateral ocellar diameters as seen in frontal view and ca 3 as seen in dorsal view; antennae attain mesoscutellum, flagellomeres (from F2 onward) 1.2-1.3 times as long as wide, tyloids well developed, covering entire lateral flagellar surface and peripheral part of ventral one (with medial glabrous spot); ocello-ocular area areolate; face with dense plumose pubescence, below antennal sockets obscuring integument. Mesoscutum areolate-punctate $(30-50 \mu \mathrm{m})$, mesoscutellum areolate with a few interspaces of at most a puncture diameter; propodeal triangle (metapostnotum) shorter than mesoscutellum, roughly reticulate-rugose; mesepisternum coarsely reticulate-rugose; legs red-brown. Metasomal terga densely punctate (15-25 $\mu \mathrm{m} / 0.5-3)$, T1 marginal zone punctured barely finer than on disc, impunctate along posterior margin; T2-T5 marginal zones impunctate; T1 red apically, T2-T3 mostly red.

\section{Female}

Unknown.

\section{Distribution}

India (Uttarakhand, Meghalaya).

Sphecodes iridipennis Smith, 1879

Fig. $11 \mathrm{~A}-\mathrm{E}$

Sphecodes iridipennis Smith, 1879: 27-28, ․

Sphecodes iridipennis - Gupta 2013: 60 .

\section{Diagnosis}

This species is close to the $S$. simlaensis owing to a similar structure, sculpture of the body and the fact that the females morphologically are difficult to distinguish, but $S$. iridipennis has a slightly less transverse head (1.1 times as wide as long vs 1.2) and entirely red legs (at least femora black or brownish in S. simlaensis).

\section{Material examined}

Holotype

INDIA• 1 \%; “Type // N Ind [Northern India] // B.M.TYPE HYM.17a550”; NHMUK 013380317.

\section{Descriptive notes}

Wings with weak yellowish or brownish darkening; hind wing with basal vein strongly curved with the angle between basal $(M)$ and cubital $(\mathrm{Cu})$ veins ca $90^{\circ}$, costal margin with five hamuli. Preoccipital carina absent. 


\section{Female}

Total body length $4.5 \mathrm{~mm}$ (Fig. 11A). Head weakly transverse, at most 1.1 times as wide as long (Fig. 11B); vertex not elevated as seen in frontal view; F1 and F2 transverse, $0.5-0.6$ times as long as wide, F3 0.8 times as long as wide; clypeus with punctures separated by 1-3 puncture diameters; ocello-ocular area with fine punctures separated by 1-3 puncture diameters; face and gena with sparse
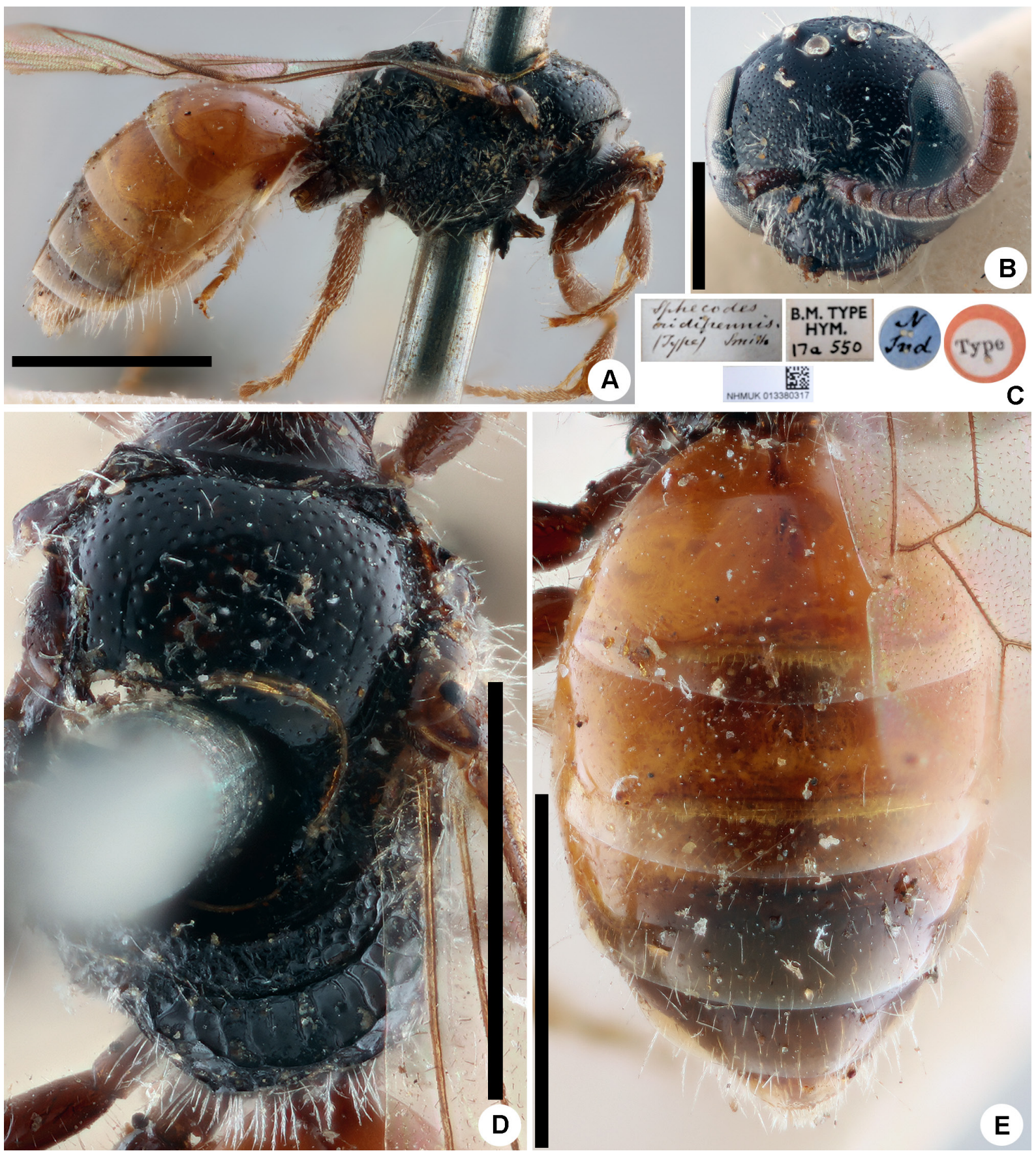

Fig. 11. Sphecodes iridipennis Smith, 1879. Holotype, $q$ (NHMUK 013380317). A. Habitus in lateral view. B. Head in frontal view. C. Labels. D. Mesosoma in dorsal view. E. Metasoma in dorsal view. Scale bars: A, D-E $=1 \mathrm{~mm} ; \mathrm{B}=0.5 \mathrm{~mm}$. 
pubescence. Mesoscutum and mesoscutellum with punctures (15-20 $\mu \mathrm{m})$ separated by 1-4 puncture diameters (Fig. 11D); hypoepimeral area coarsely reticulate; metafemur weakly enlarged in proximal half, maximum width 0.35 times its length; legs red. Propodeal triangle (metapostnotum) with coarse longitudinal wrinkles and shiny interspaces. Metasomal terga scarcely punctate, T1 impunctate, remaining terga basally with sparse fine setal pores (Fig. 11E); marginal zones impunctate; terga red to red-brownish apically, pygidial plate 0.7 times as wide as metabasitarsus.

Male

Unknown.

\section{Distribution}

Himalayas: Uttarakhand, Northern India. The record of the species from Himachal Pradesh (India) by Saini \& Rathor (2012: 162) is doubtful.

\section{Remarks}

Since we could not clearly differentiate between females of S. iridipennis and S. simlaensis, except by their relative head length and coloration, more specimens should be studied (including the male finding) to make a decision on their synonymy.

The specimens recorded as $S$. iridipennis from Chapra (India) by Rajkumar \& Dey (2016: 1847, pl. 3 ) belong to another species. Unlike the holotype this specimen, re-described as S. iridipennis, has more transverse head (1.3 times as wide as long) and possibly belongs to the undescribed female of S. chaprensis Blüthgen, 1927.

Sphecodes kershawi Perkins, 1921

Figs 2G, 5D

Sphecodes kershawi Perkins, 1921: 9, ठ̂.

Sphecodes javanensis Blüthgen, 1927: 69-70, §. Synonymized by Astafurova et al. 2020b: 62.

\section{Diagnosis and descriptive notes}

See Astafurova et al. (2020b: 63-64).

\section{Material examined}

\section{Syntypes}

CHINA 1 1 , syntype; China, Macao, J.C. Kershaw leg.; NHMUK $\bullet 1 \hat{\jmath}$, syntype; same collection data as for preceding; ZMHB.

\section{Other material}

INDIA • 1 क; Uttarakhand, Uttarkashi; $30^{\circ} 73^{\prime}$ N, $78^{\circ} 45^{\prime}$ E; 1235 m a.s.l.; 25 Apr.-2 May 2012; K. Tomkovich leg.; ZMMU.

INDONESIA 1 § , holotype of Sphecodes javanensis Blüthgen, 1927; Java, Buitenzorg; Aug. 1920; NHMW.

\section{Distribution}

Himalayas: India (*Uttarakhand); China (Macao), Myanmar, Malaysia. 
Sphecodes lasimensis Blüthgen, 1927

Figs $12,13 \mathrm{~A}-\mathrm{C}$

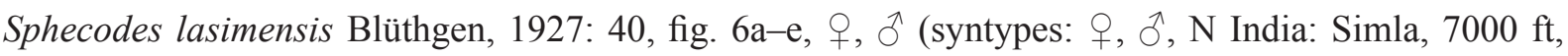
Annandale leg. 16./5/1909; Simla Hills, 9000 ft, Annandale u. Kemp leg. 18-21./5.1916; ?The Indian Museum, Calcutta; not examined).

Sphecodes lasimensis - Ascher \& Pickering 2020: map.

\section{Diagnosis}

The female is closest to that of $S$. gibbus by the shape of the head and sculpture of the body, but differs from this species by the less elevated vertex with distance from top of head to upper margin of lateral ocellus approximately two lateral ocellar diameters as seen in frontal view (vs ca 3), the sparser punctate metasoma with almost impunctate marginal zones on T1 and T2 (distinctly punctate in S. gibbus) and on average smaller body length $7.5-10 \mathrm{~mm}$ (vs $7-15 \mathrm{~mm}$ ). We have not studied the male, but according to the description of Blüthgen (1927) it is similar to that of the Palaearctic S. alternatus Smith, 1853 and S. reticulatus Thomson, 1870 owing to similar sculpture, shapes of antennal tyloid area and gonostylus (tyloids of $S$. lasimensis are more developed and semicircular across basal $1 / 5-1 / 3$ of ventral flagellar surface vs $1 / 7-1 / 5$ in $S$. alternatus and $S$. reticulatus).

\section{Material examined}

NEPAL $• 2$ 우; Kali-Gandaki-Kola, Tatopani, 50 km SW of Pokhara; 1100-1400 m a.s.1.; 12-15 May 1984; C. Holzschuh leg.; PCMS.

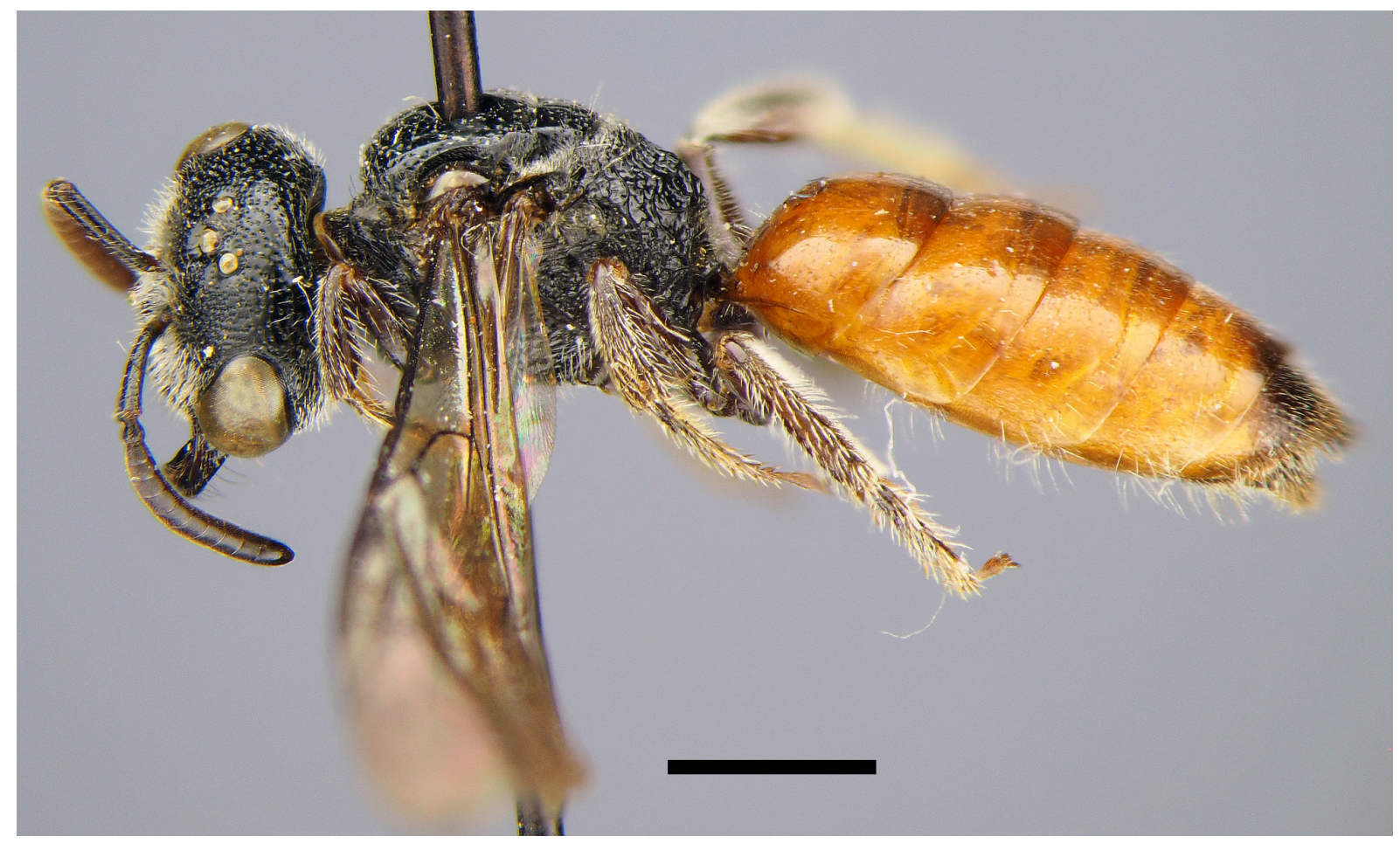

Fig. 12. Sphecodes lasimensis Blüthgen, 1927, $\uparrow(\mathrm{PCMS})$. Habitus in lateral view. Scale bar $=1 \mathrm{~mm}$. 


\section{Descriptive notes}

Wings with brownish darkening; hind wing with the angle between basal $(M)$ and cubital $(\mathrm{Cu})$ veins ca $70^{\circ}$, costal margin with eight, nine or ten hamuli.

\section{Female}

Total body length 7.5-10 mm (Fig. 12). Head transverse, ca 1.25 times as wide as long (Fig. 13A); vertex elevated with distance from top of head to upper margin of lateral ocellus approximately two lateral ocellar diameters as seen in frontal view; labrum trapezoidal, 0.7 times as long as basal width; ocello-ocular area and vertex irregularly punctate with punctures separated by $0.5-4$ puncture diameters;
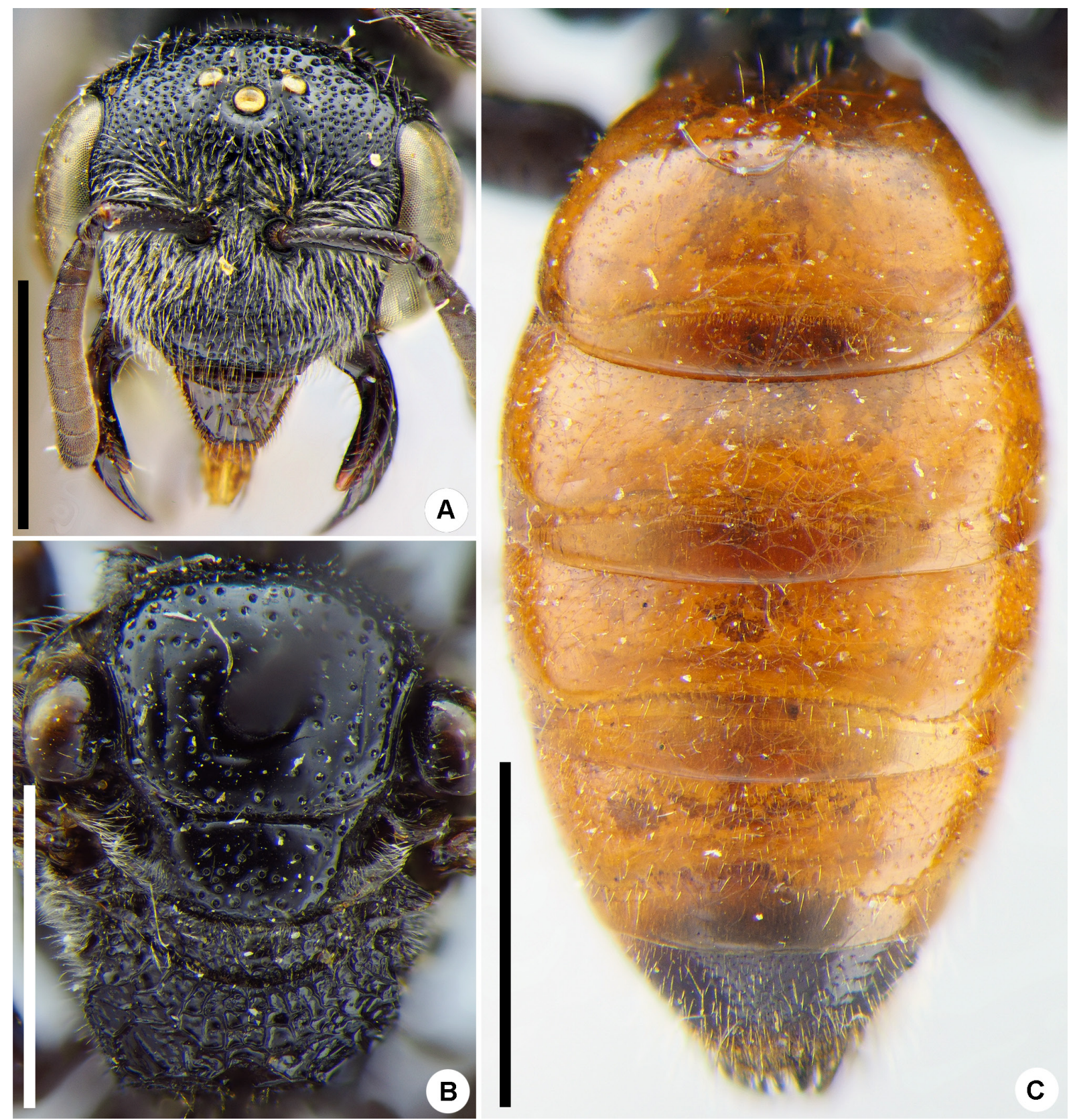

Fig. 13. Sphecodes lasimensis Blüthgen, 1927, o (PCMS). A. Head in frontal view. B. Mesosoma in dorsal view. C. Metasoma in dorsal view. Scale bars $=1.0 \mathrm{~mm}$. 
paraocular area with dense adpressed white pubescence, but not obscuring integument, gena with sparser pubescence. Mesoscutum and mesoscutellum sparsely punctate $(30-50 \mu \mathrm{m} / 1-7)$; propodeal triangle (metapostnotum) and mesepisternum reticulate-rugose (Fig. 13B). Metasomal terga sparsely punctate, on discs with shallow tiny punctures separated by a few diameters (Fig. 13C); T1 marginal zone indistinctly punctate with a few tiny punctures; T2-T4 marginal zone smooth and impunctate; pygidial plate 0.5 times as wide as metabasitarsus; T1-T4 red, T5 partially brownish.

Male (according to Blüthgen 1927)

Head weakly transverse, ca 1.15 times as wide as long; vertex elevated with distance from top of head to upper margin of lateral ocellus approximately two lateral ocellar diameters as seen in frontal view. Tyloids (from F4 onward) weakly developed, semicircular across basal $1 / 5-1 / 3$ of ventral surface of flagellomeres.

\section{Distribution}

Himalayas: India (Himachal Pradesh), *Nepal.

Sphecodes laticeps Meyer, 1920

Fig. 5B

Sphecodes laticeps Meyer, 1920: 121, ㅇ, ðิ.

Sphecodes candidius Meyer, 1925: 10, ․ Synonymized by Blüthgen 1927: 85.

Sphecodes biroi mariae Cockerell, 1930: 162, ô (holotype: đ., Thailand, "Siam, Nam, Jan. 8, 1928

(Cockerell)"; USNM, http://n2t.net/ark:/65665/3e3daca86-a75f-458d-b994-6723b995dccd). Synonymized by Astafurova et al. 2020b: 65 .

\section{Diagnosis and descriptive notes}

See Astafurova et al. (2020b: 65-67).

\section{Material examined}

NEPAL・ 1 đ̊; Bagmati; 1600-2000 m a.s.1.; 26 Aug. 1975; Takagi-S. leg.; PCMS.

TAIWAN 11 , lectotype (designated by Astafurova et al. 2020b: 64); Formosa [Taiwan], Takao; ZMHB • , , holotype of Sphecodes candidius Meyer, 1925; "Lake Candidius 25./9/-10./10/ 1907”; HNHM.

\section{Distribution}

Himalayas: *Nepal; China (Yunnan, Taiwan), Thailand, Vietnam.

\section{Sphecodes monilicornis (Kirby, 1802)}

Figs 3B, 5C

Melitta monilicornis Kirby, 1802: 47, ô (type locality: England).

Sphecodes maculatus Lepeletier de Saint Fargeau, 1841: 545, ठิ (type locality: France).

Sphecodes subquadratus Smith, 1845: 1014, + , § (type locality: England).

Sphecodes gibbus var. ephippium subvar. rufipes Sichel, 1865: 428, +, §ิ. Unavailable name (ICZN 1999, Article 45.5 of Code).

Sphecodes gibbus var. ephippium subvar. dubius Sichel, 1865: 419, §̊. Unavailable name (ICZN 1999, Article 45.5 of Code).

Sphecodes gibbus var. ephippium subvar. incertus Sichel, 1865: 420, §ิ. Unavailable name (ICZN 1999, Article 45.5 of Code). 
Sphecodes gibbus var. ephippium subvar. nigrescens Sichel, 1865: 427, §. Unavailable name (ICZN 1999, Article 45.5 of Code).

Sphecodes gibbus var. ephippium subvar. testaceipes Sichel, 1865: 428, đ. Unavailable name (ICZN 1999, Article 45.5 of Code).

Sphecodes ruficrus Dalla Torre, 1896: 9 (nom. praeocc., nec Erichson, 1835), replacement name for Sphecodes rufipes Sichel, 1865.

Sphecodes hanuman Nurse, 1903: 538, ㅇ (syntypes: 우 을 India, Jammu and Kashmir, 5-6000 ft, 5.01; NHMUK; not examined). Synonymized by Blüthgen 1927: 39.

Sphecodes caucasicus Meyer, 1920: 124, §̋ (type locality: Georgia).

Sphecodes cephalotes Meyer, 1920: 129, o (type locality: Turkey).

Sphecodes smyrnensis Meyer, 1920: 116, ठ (type locality: Turkey).

Sphecodes monilicornis quadratus Meyer, 1920: 129, ô, ㅇ (type locality: Spain).

Sphecodes monilicornis var. nigerrima Blüthgen, 1927: 41, ô (syntypes: 3 ô $\widehat{~}$, Pakistan: Quetta; India:

Sringar (Jammu and Kashmir); ?ZMHB; not examined). Synonymized by Warncke 1992: 22.

Sphecodes quadratus cephalotiformis Pittoni, 1950: 62, ô, o (type locality: Cyprus).

Sphecodes monilicornis berberus Warncke, 1992: 22, ô, ㅇ (type locality: Morocco).

Sphecodes monilicornis - Ascher \& Pickering 2020: map.

\section{Diagnosis}

See Astafurova et al. (2018a: 24).

\section{Material examined}

NEPAL・ 1 क; Godavari, Napal Valley; 26 Mar. 1968; T. Matsumura leg.; PCMS.

\section{Distribution}

Himalayas: India (Jammu and Kashmir), *Nepal; North Africa, Europe (north to $64^{\circ}$ ), Turkey, Caucasus, Jordan, Syria, Kazakhstan, Central Asia, Iran, Pakistan, Russia (east to Far East), Mongolia, China (Heilongjiang).

Sphecodes montanus Smith, 1879

Figs 3C, 4A-B

Sphecodes montanus Smith, 1879: 27, ㅇ, §ิ.

Sphecodes montanus - Gupta 2013: 60. — Ascher \& Pickering 2020: map.

\section{Diagnosis and descriptive notes}

See Astafurova et al. (2020b: 68-70).

\section{Material examined}

\section{Syntype}

INDIA • 1 q; "Type // N.Ind // Masuri [Mussoorie, Uttarakhand, India] 7000 feet // Pres. by Imp. Inst. Ent. Brit. Mus. 1932-360 // Sphecodes montanus Smith, Type ô et + // B.M.Type HYM.17a549”; NHMUK 013380316.

\section{Other material}

NEPAL • 1 \%; Jumla Distr.; 12-14000 ft a.s.l; May-Jun. 1961; J. Burmet leg.; NHMUK 013380477 • 1 †, 1 §̊; Godavari, Napal Valley; 26 Mar. 1968; T. Matsumuta leg.; PCMS • 1 क; Gosainkund; 18 Feb. 1968; 8000 ft a.s.1.; T. Kawamichi leg.; PCMS • 2 우; Koshi Basantapur; 2300 m a.s.1.; 30 May-2 
Jul. 1985; C. Holzschuh leg.; PCMS • 1 q; Rasuwa Distr., Langtang NP, Dhunche-Bharkhu-Syabru; 2000-2800 m a.s.1.; 6-13 May 1996; P. Cechovski leg.; PCMS • 1 +; Terhatum Distr., Tamur Valley, Basantpur; 2400 m a.s.1.; 29-30.V.1996; P. Cechovski leg.; PCMS • 1 क; NE Kathmandu, Nagar Kot; 1800-2000 m a.s.1.; 26 Jun. 1980; C. Holzschuh leg.; PCMS.

INDIA - 2 + $\circ$; Uttarakhand, Uttarkashi; $30^{\circ} 73^{\prime} \mathrm{N}, 78^{\circ} 45^{\prime} \mathrm{E} ; 1235 \mathrm{~m}$ a.s.1.; 1 May-25 Jun. 2012; K. Tomkovich leg.; ZMMU.

BHUTAN • 1 क; Timphu; 10 Mar. 1985; D.E. Padgham leg.; NHMUK 013380472.

\section{Distribution}

Himalayas: India (Uttarakhand), *Nepal, *Bhutan; India (Rajasthan), Laos, China (Xizang, Sichuan, Yunnan).

\section{Sphecodes perplexus Nurse, 1903}

Figs 14, 15A-D

Sphecodes perplexus Nurse, 1903: 540, ㅇ.

Sphecodes perplexus - Saini \& Rathor 2012: 163. — Ascher \& Pickering 2020: map.

\section{Diagnosis}

The female of this species is similar to that of $S$. tantalus Nurse, 1903 owing to a similar structure, sculpture and coloration of the body, but it differs from this species by having a more densely punctate

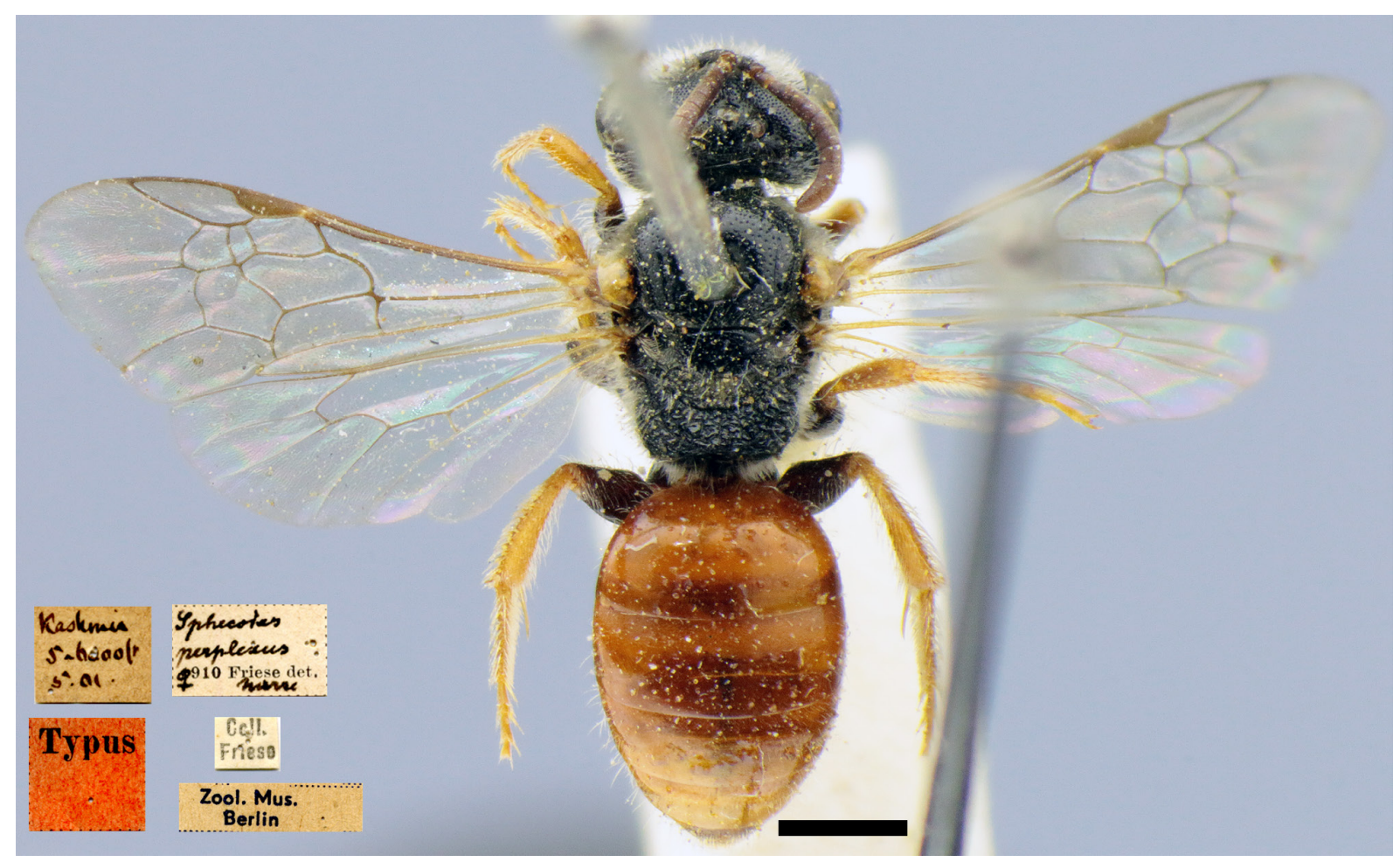

Fig. 14. Sphecodes perplexus Nurse, 1903. Syntype, $q$ (ZMHB). Habitus in dorsal view and labels. Scale bar $=1 \mathrm{~mm}$. 

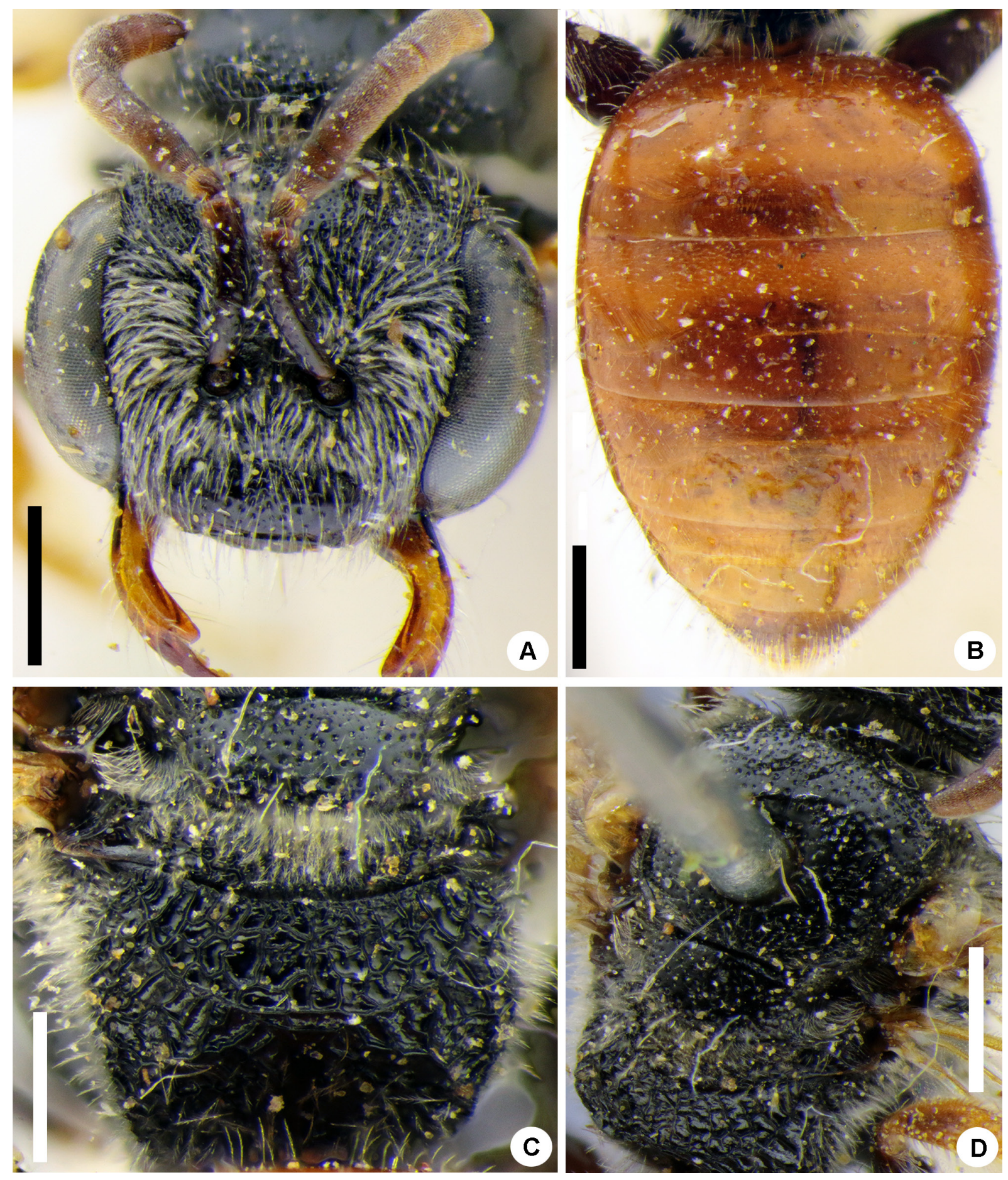

Fig. 15. Sphecodes perplexus Nurse, 1903. Syntype, $q$ (ZMHB). A. Head in frontal view. B. Metasoma in dorsal view. C. Mesoscutum and propodeum in dorsal view. D. Mesosoma in dorsal view. Scale bars $=0.5 \mathrm{~mm}$. 
mesoscutum, with the punctures separated by 1-2 puncture diameters (vs $2-5$ ), and the pygidial plate as wide as the metabasitarsus (vs 0.8). Both these species are close to $S$. crassus Thomson, 1870 by the strongly enlarged metafemur, but it differs by the entirely red metasoma.

\section{Material examined}

\section{Syntypes}

INDIA • 1 O; "syntype: <red circle>", "Kashmir 5-6000 ft. 5.01", "Col. C. G. Nurse Collection, 192072", "Sphecodes perplexus Nurse", "B.M. TYPE HYM. 17.a.586"; NHMUK • 1 क; "Kashmir 5-6000 ft. 5.01", "Sphecodes perplexus Friese det.", "Coll. Friese", "Typus"; ZMHB (label photo Fig. 14).

\section{Descriptive notes}

Wings hyaline, without darkening; hind wing with the angle between basal $(M)$ and cubital $(\mathrm{Cu})$ veins ca $80^{\circ}$, costal margin with five hamuli.

\section{Female}

Total body length $6.5 \mathrm{~mm}$ (Fig. 14). Head (Fig. 15A) strongly transverse, ca 1.3 times as wide as long; vertex weakly elevated as seen in frontal view (distance from top of head to upper margin of lateral ocellus at most a lateral ocellar diameter), but wide as seen in dorsal view (approximately three ocellar diameters); F1 and F2 strongly transverse, ca 0.6 times as long as wide, F3 weakly transverse, 0.8 times as long as wide; labrum semi-oval, 0.6 times as long as wide; clypeus and ocello-ocular area with dense punctures separated by $0.5-1$ puncture diameter; paraocular and supracypeal areas with dense plumose pubescence, but not obscuring integument. Mesoscutum (Fig. 15C-D) with sparse punctures $(20-25 \mu \mathrm{m})$ separated by one or two puncture diameters; hypoepimeral area reticulate; metafemur strongly enlarged in proximal half, maximum width 0.5 times its length; tibia and tarsi red-yellowish. Metasomal T1 impunctate (Fig. 15B), T2 on an anterior half with fine and relatively dense punctures; marginal zones impunctate; pygidial plate as wide as metabasitarsus; T1-T5 red.

\section{Male \\ Unknown.}

\section{Distribution}

Himalayas: India (Jammu and Kashmir).

\section{Sphecodes setiger Blüthgen, 1924}

Fig. 16A-D

Sphecodes setiger Blüthgen, 1924: 511-512, q.

Sphecodes setiger - Ascher \& Pickering 2020: map.

\section{Diagnosis}

This species is similar to small Palaearctic species with simple mandibles which lack the inner tooth (i.e., Sphecodes decorus (Cameron, 1897), S. longuloides Blüthgen, 1923, S. hirtellus Blüthgen, 1923, S. longulus Hagens, 1882, S. puncticeps Thomson, 1870, S. turanicus Astafurova \& Proshchalykin, 2017 and S. trjapitzini Astafurova \& Proshchalykin, 2018). Among these species S. setiger is closest to $S$. puncticeps, but differs from this species by having square (as long as wide) F1 and F2 (vs 0.60.7 times as long as wide). 


\section{Material examined}

\section{Holotype}

INDIA • +; “Type // Simla [India], Nurse 9. 98 // Col. C.G. Nurse Collection. 1920-72 // Sph. setiger,, , Type P. Blüthgen det. // B.M.Type HYM.17a562"; NHMUK 013380328.

\section{Descriptive notes}

Wings hyaline, without darkening; hind wing with basal vein strongly curved with the angle between basal $(M)$ and cubital $(C u)$ veins almost $90^{\circ}$, costal margin with five hamuli.

\section{Female}

Total body length $5.5 \mathrm{~mm}$. Head strongly transverse, 1.25 times as wide as long (Fig. 16A); vertex not elevated; F1-F3 square, as long as wide (Fig. 16B); clypeus with punctures separated by at most
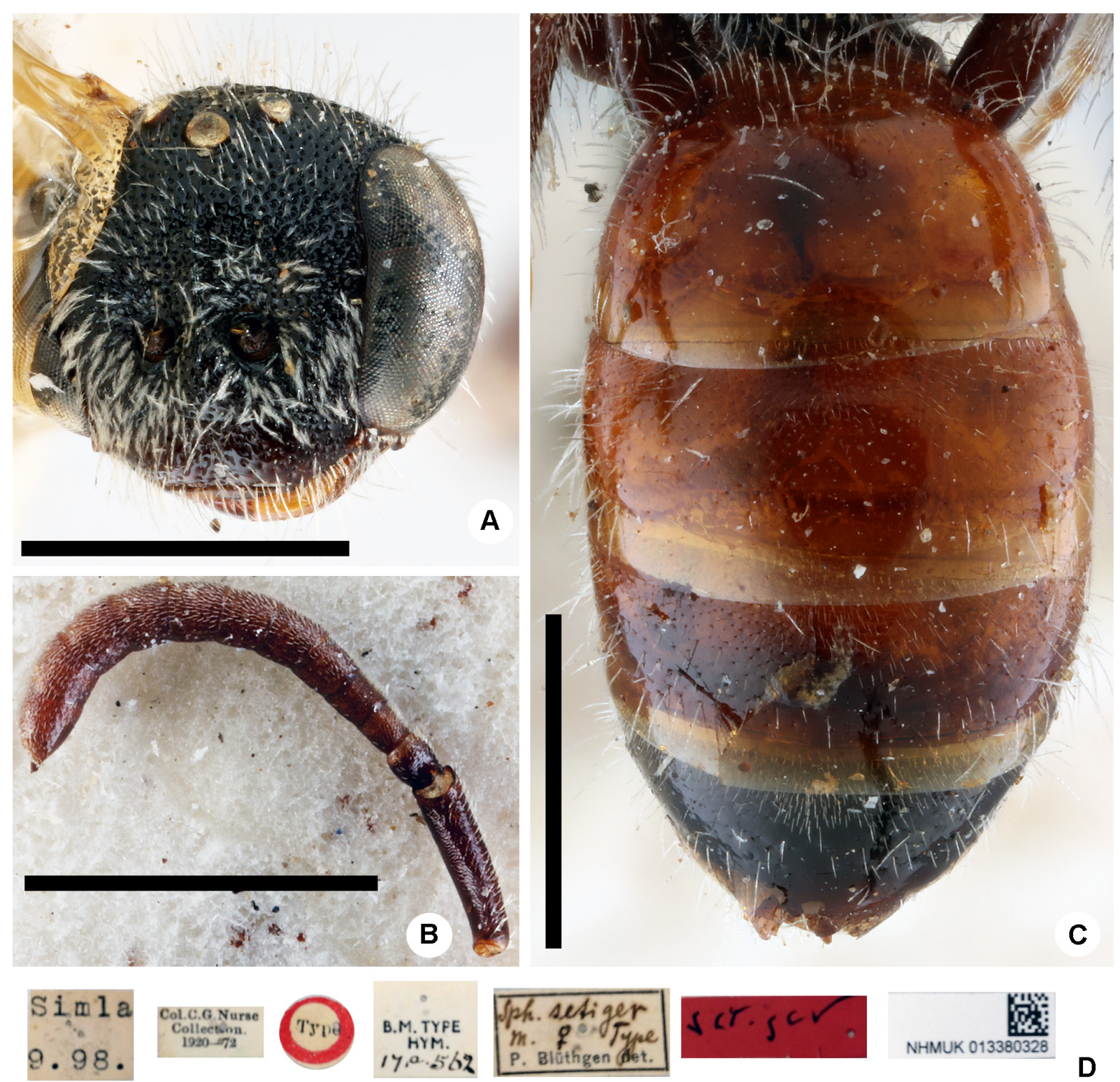

Fig. 16. Sphecodes setiger Blüthgen, 1924. Holotype, q (NHMUK 013380328). A. Head in frontal view. B. Antenna in lateral view. C. Metasoma in dorsal view. D. Labels. Scale bars $=1 \mathrm{~mm}$. 
a puncture diameter; ocello-ocular area with dense punctures separated by at most a half puncture diameter; paraocular and supraclypeal areas with relatively dense plumose pubescence, but not obscuring integument. Mesoscutum with punctures $(15-30 \mu \mathrm{m})$ separated by at most a puncture diameter; hypoepimeral area coarsely reticulate; legs red-brownish. Metasomal T1 impunctate, except a few punctures $(5 \mu \mathrm{m})$ along marginal zone; anterior half of $\mathrm{T} 2-\mathrm{T} 4$ discs distinctly punctate (10$15 \mu \mathrm{m} / 2-5$ ), sparser on posterior half; T1-T3 red (Fig. 16C); pygidial plate as wide as metabasitarsus.

\section{Male \\ Unknown.}

\section{Distribution}

Himalayas: India (Himachal Pradesh).

\section{Remarks}

Since we could not clearly differentiate between females of $S$. setiger and $S$. puncticeps, except by relative flagellar length, more specimens from the type locality including the male are needed to be studied to make a decision on their synonymy.

\section{Sphecodes shillongensis Blüthgen, 1927}

Fig. 17A-D

Sphecodes shillongensis Blüthgen, 1927: 95, fig. 29a-b.

Sphecodes shillongensis - Saini \& Rathor 2012: 163. — Ascher \& Pickering 2020: map.

\section{Diagnosis}

This species is similar to $S$. chaprensis and S. simlaensis, sharing similar shape and sculpture of the body, including weakly developed antennal tyloids, a densely punctate mesoscutum and scarcely punctate metasomal terga, but differs from these species by having longer antennae with flagellomeres (from F3 onward) ca 1.3 times as long as wide (vs 1.1-1.2) and the shape of the gonostylus without a membranous part.

\section{Material examined}

\section{Holotype}

INDIA • Õ; "Type // Shillong, 4.03 // Assam, R. Turner. 1905-125. // Sphecodes shillongensis n.sp., Ô, P. Blüthgen det. // B.M.Type HYM.17a.563"; NHMUK 013380326.

\section{Descriptive notes}

Wings hyaline; hind wing with the angle between basal $(M)$ and cubital $(\mathrm{Cu})$ veins almost $90^{\circ}$, costal margin with five hamuli. Preoccipital carina absent.

\section{Male}

Total body length $5.0 \mathrm{~mm}$. Head transverse, ca 1.15 times as wide as long (Fig. 17A); vertex not elevated as seen in frontal view; antennae attaining posterior margin of mesoscutellum, F1 0.75 times as long as wide, remaining flagellomeres ca 1.3 times as long as wide; tyloids weakly developed, covering at most basal $1 / 5-1 / 4$ of ventral surface of last flagellomeres (Fig. 17A); ocello-ocular area with dense punctures $(10-15 \mu \mathrm{m})$ separated by at most a half puncture diameter; face with dense plumose white pubescence, obscuring integument below antennal sockets. Gena with sparser pubescence. Mesoscutum and mesoscutellum finely punctate, sparser medially (15-20 $\mu \mathrm{m} / 0.5-3)$, becoming denser peripherally; 
mesepisternum and hypoepimeral area rugose; propodeal triangle (metapostnotum) shining, with coarse longitudinal wrinkles; remaining surfaces of propodeum reticulate-rugose (Fig. 17C); legs red. Metasoma red; terga scarcely punctate (Fig. 17D); T1 almost impunctate, with a few microscopic punctures (3-5 $\mu \mathrm{m})$; remaining terga basally with tiny setal pores; marginal zones impunctate; gonocoxite dorsally with impression; gonostylus small, without a membranous part.
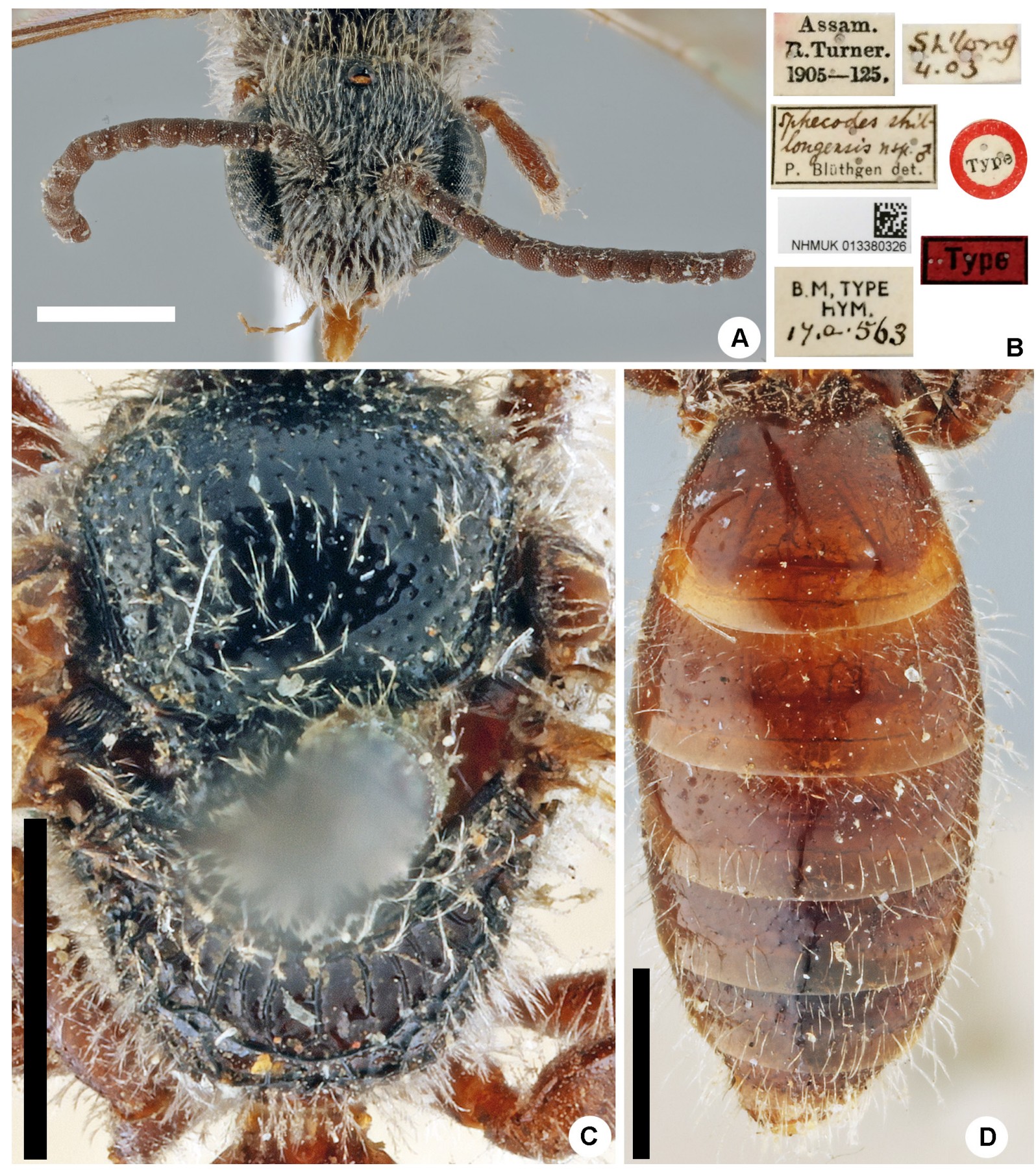

Fig. 17. Sphecodes shillongensis Blüthgen, 1927. Holotype, ठ̊ (NHMUK 013380326). A. Head in frontal view. B. Labels. C. Mesosoma in dorsal view. D. Metasoma in dorsal view. Scale bars $=0.5 \mathrm{~mm}$. 
Female

Unknown.

\section{Distribution}

Himalayas: India (Meghalaya).

Sphecodes sikkimensis Blüthgen, 1927

Fig. 18A-E

Sphecodes sikkimensis Blüthgen, 1927: 54, fig. 12a, ․ .

Sphecodes sikkimensis - Ascher \& Pickering 2020: map.

\section{Diagnosis and descriptive notes}

See Astafurova et al. (2020b: 78) and refer to the diagnosis of Sphecodes uttaricus sp. nov. below.

\section{Material examined}

Lectotype (designated here)

INDIA • 1 क; "Sikhim [India], 6.97., Rungit Tal, 1000', Coll. Bingham // Sphec. fumippenis Dr. Meyer det. // Sphec. sikkimensis n.sp. P. Blüthgen det.”; ZMHB.

\section{Paralectotypes}

INDIA $\bullet 2$ + 9 ; same collection data as for lectotype (same labels); ZMBH.

\section{Distribution}

Himalayas: India (Sikkim); Myanmar, Laos, China (Sichuan, Yunnan, Fujian, Guandong).

\section{Sphecodes simlaensis Blüthgen, 1924}

Fig. $3 \mathrm{G}$

Sphecodes simlaensis Blüthgen, 1924: 514-515, ㅇ (syntypes: 2 우, India, Simla, Nurse leg., VIII. and IX. 98; were not found in NHMUK).

Sphecodes simlaellus Blüthgen, 1927: 46-48, fig. 8, §̂. Synonymized by Astafurova et al. 2020b: 79.

Sphecodes simlaensis - Ascher \& Pickering 2020: map.

\section{Diagnosis and descriptive notes}

See Astafurova et al. (2020b: 79-81).

\section{Material examined}

INDIA $-2 \lesssim え$, lectotype and paralectotype of S. simlaellus, designated by Astafurova et al. (2020b: 79); "Simla [India, Himachal Pradesh], 8.[18]98, Col. C.G. Nurse Collection. 1920-72"; NHMUK • 6 우; Kasmir [Jammu and Kashmir]; Jul. 1901; Nurse leg.; ZMHB •; 6 q $O$; Uttarakhand, Uttarkashi; $30^{\circ} 73^{\prime}$ N, 7845' E; 1235 m a.s.1.; 1-2 May 2012; K. Tomkovich leg.; ZMMU.

NEPAL • 1 đ; Gulbu Bhanjang; 9 Jun. 1968; T. Matsumura leg.; PCMS • 1 đ; Namche Bazar; 8 Jul. 1968; T. Matsumura leg.; PCMS • 1 \%; Nangitanti; 1 May 1968; T. Matsumura leg.; PCMS • 1 q; Everest Reg., Jambesi; 2734' N, 86³2' E; 19 Mar. 2010; A. Reschikov leg.; ZISP • 2 우; Bagam, Sindhupal Chok, Sarmatang; $27^{\circ} 57^{\prime}$ N, 85 56 $56^{\prime}$ E; 2500 m a.s.l.; C. Holzschuh leg.; PCMS • 2 우; Rasuwa Distr., 

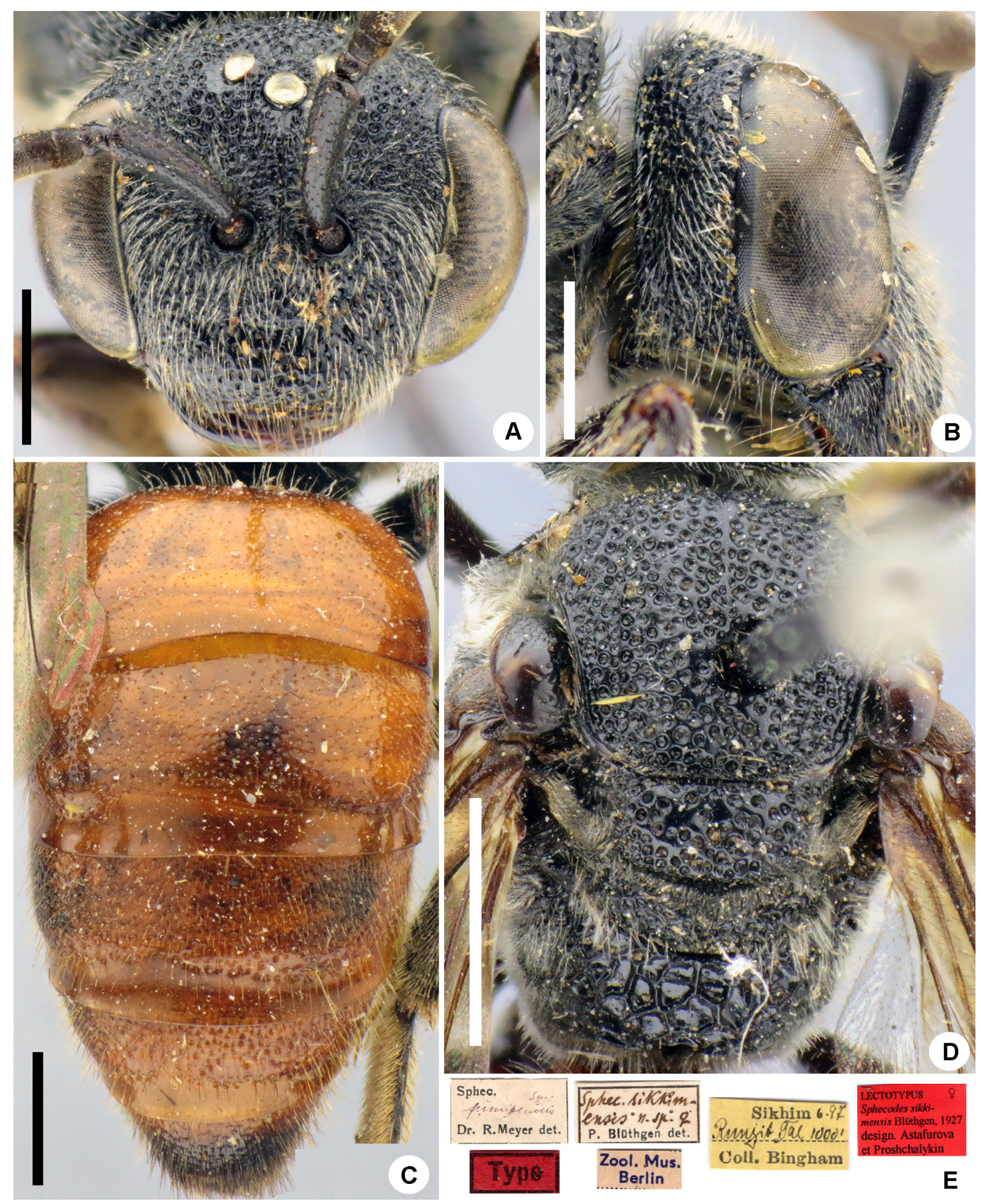

Fig. 18. Sphecodes sikkimensis Blüthgen, 1927. Lectotype, $q$ (ZMBH). A-B. Head in frontal (A) and lateral view (B). C. Metasoma in dorsal view. D. Propodeum in dorso-lateral view. E. Labels. Scale bars: $\mathrm{A}-\mathrm{C}=1 \mathrm{~mm}$; $\mathrm{D}=0.5 \mathrm{~mm}$. 
Langtang NP, Dhunche-Bharkhu-Syabru; 2000-2800 m a.s.1.; 6-13 May 1996; P. Cechovski leg.; PCMS • 1 क; Koshi Gorza; 2100 m a.s.1.; 5 Jun. 1985; C. Holzschuh leg.; PCMS.

BHUTAN • 3 + 9 ; Paro Prov., Chiley-La; 3000-3500 m a.s.l.; C. Holzschuh leg.; PCMS • 1 đ̊; Thimphu Distr., Taba; 2600 m a.s.l.; C. Holzschuh leg.; PCMS.

\section{Distribution}

Himalayas: India (Jammu and Kashmir, Himachal Pradesh, *Uttarakhand), *Nepal, *Bhutan; Laos, China (Sichuan, Yunnan).

Sphecodes tantalus Nurse, 1903

Figs 19, 20A-D

Sphecodes tantalus Nurse, 1903: 539, .

Sphecodes tantalus - Dar et al. 2018: 531. — Dar \& Wani 2018: 1432.

\section{Diagnosis}

The female of this species is similar to that of S. perplexus (refer to Diagnosis of S. perplexus above).

\section{Material examined}

\section{Syntypes}

INDIA • 1 \%; "(syntype), <red circle>”, "Kashmir [Jammu and Kashmir] 5-6000 ft. 5.01", "Col. C. G. Nurse Collection, 1920-72", "Sphecodes tantanus Nurse", “o, Type”, "B.M. TYPE HYM. 17.a.585”; NHMUK 013380356・1 o; "Kashmir 5-6000 ft. 5.01”; ZMHB.

\section{Descriptive notes}

Wings hyaline, without brownish darkening; hind wing with the angle between basal $(M)$ and cubital $(\mathrm{Cu})$ veins ca $80^{\circ}$, costal margin with six hamuli.

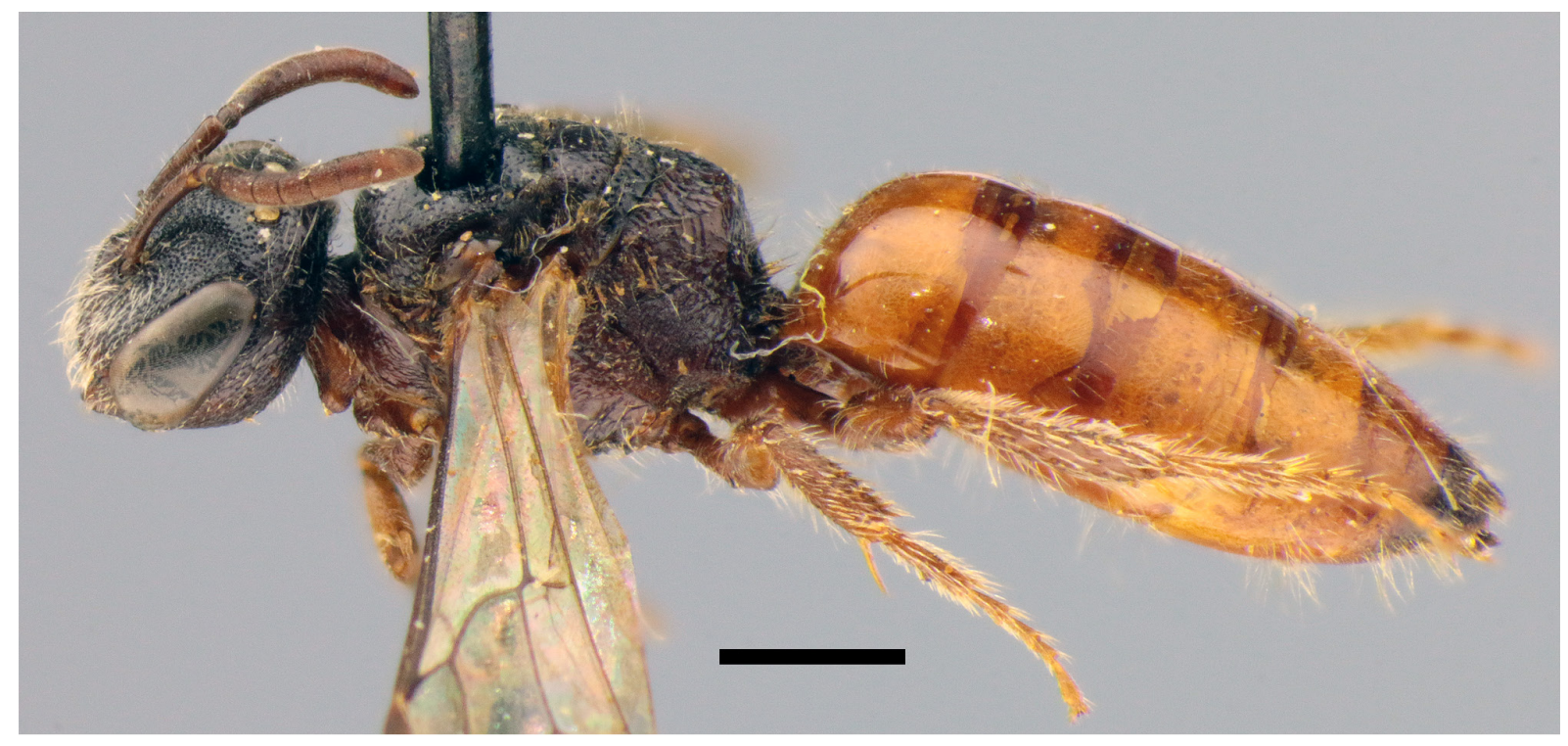

Fig. 19. Sphecodes tantalus Nurse, 1903. Syntype, $q$ (ZMHB). Habitus in lateral view. Scale bar $=$ $1 \mathrm{~mm}$. 

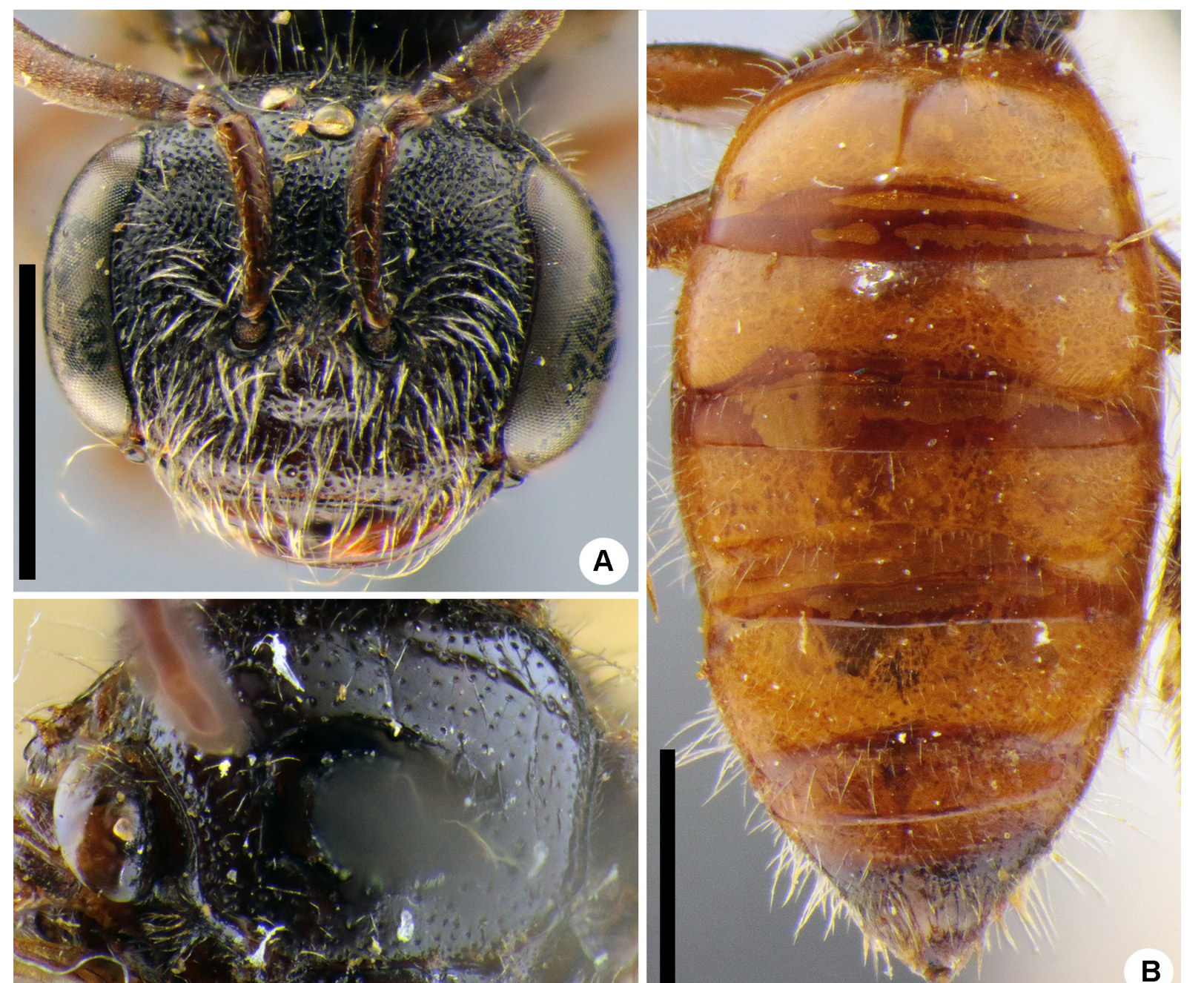

A

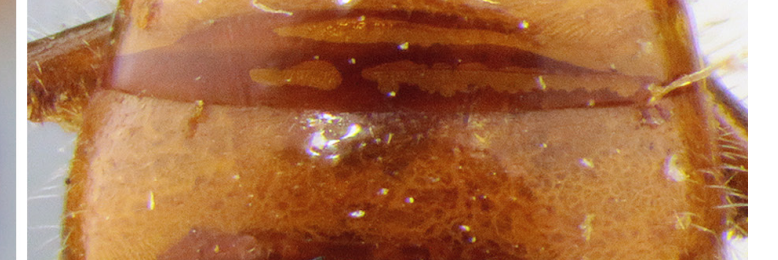




\section{Female}

Total body length $8 \mathrm{~mm}$ (Fig. 19). Head strongly transverse, ca 1.3 times as wide as long (Fig. 20A); vertex weakly elevated as seen in frontal view (distance from top of head to upper margin of lateral ocellus at most a lateral ocellar diameter), but wide as seen in dorsal view (approximately three ocellar diameters); F1 and F2 strongly transverse, 0.6-0.7 times as long as wide, F3 almost square; labrum semi-oval, 0.6 times as long as wide; clypeus and ocello-ocular area with dense punctures separated by at most a half puncture diameter; paraocular and supracypeal areas with relatively dense, but thin plumose pubescence, not obscuring integument. Mesoscutum with sparse punctures $(20-25 \mu \mathrm{m})$ separated by $2-5$ puncture diameters (Fig. 20C); hypoepimeral area reticulate; metafemur strongly enlarged in proximal half, maximum width 0.4 times its length; legs red. Metasomal T1 impunctate, T2-T4 with a few fine setal pores, marginal zones impunctate; pygidial plate dull, 0.8 times as wide as metabasitarsus; T1-T5 red (Fig. 20B).

\section{Male \\ Unknown.}

\section{Distribution}

Himalayas: India (Jammu and Kashmir); India (Rajasthan according to Ascher \& Pickering 2020: map).

\section{Description of a new species from India}

Sphecodes uttaricus sp. nov. urn:lsid:zoobank.org:act:A059C88D-0961-420A-9C97-FC19D1D2248D

Figs 21, 22A-E

\section{Diagnosis}

This species is closest to $S$. sikkimensis and S. formosanus Cockerell, 1911 by a combination of large body size (more than $9 \mathrm{~mm}$ ), costal margin with at least nine hamuli and the presence of a lateral

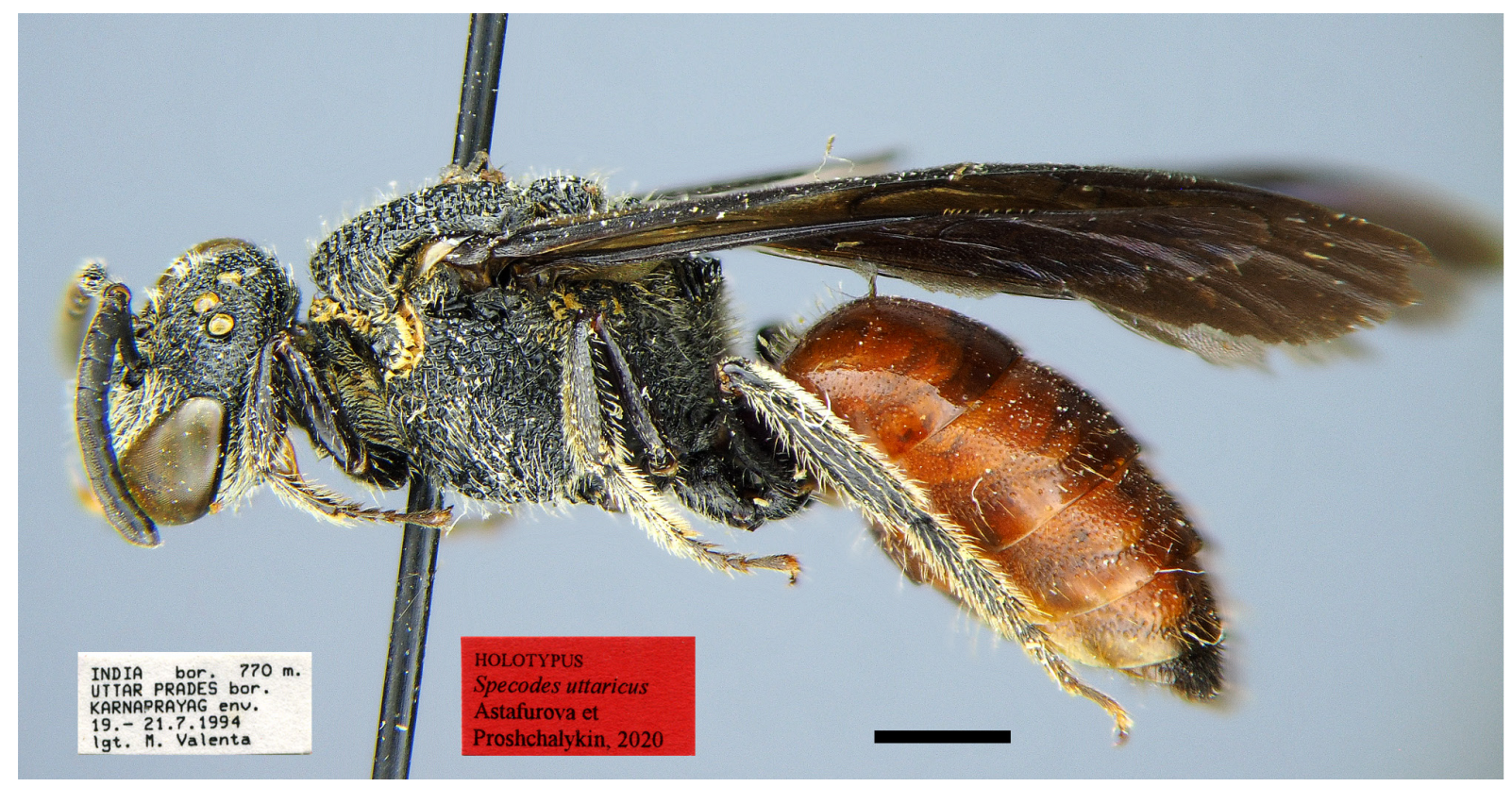

Fig. 21. Sphecodes uttaricus sp. nov. Holotype, $q$ (PCMS/OLBL). Habitus in lateral view and labels. Scale bar $=1 \mathrm{~mm}$. 

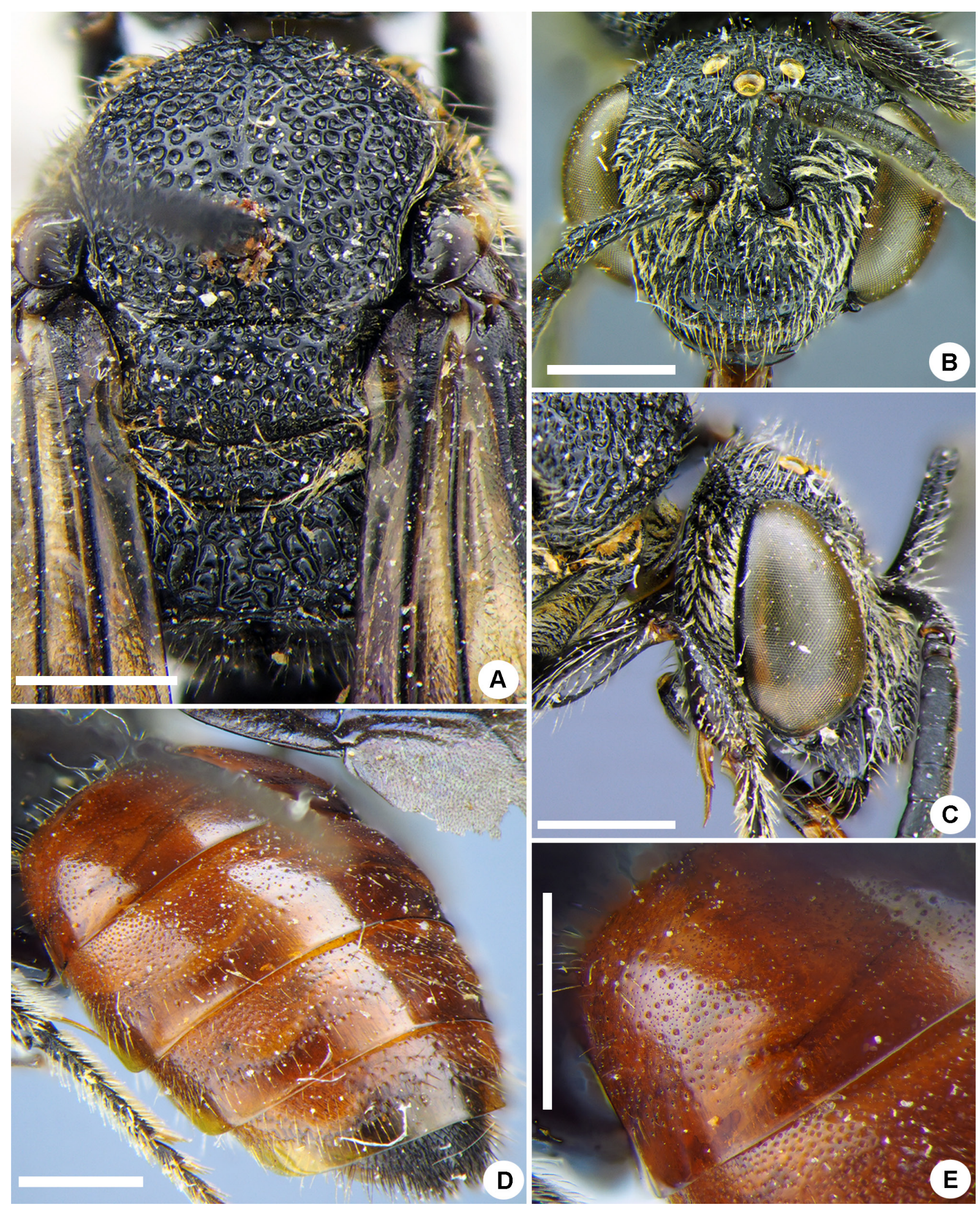

Fig. 22. Sphecodes uttaricus sp. nov. Holotype, $q$ (PCMS/OLBL). A. Mesosoma, dorsal view. B-C. Head in frontal (B) and lateral view (C). D. Metasoma in dorso-lateral view. E. T1 in dorso-lateral view. Scale bars $=1 \mathrm{~mm}$. 
preocipital carina. The new species differs from S. sikkimensis by having fewer hamuli (9-10 vs 12-15) and a smaller body size $(10.5 \mathrm{~mm}$ vs $12-15 \mathrm{~mm})$ and from $S$. formosanus it differs by having sparse and mixed punctation of T1 with coarse (15-25 $\mu \mathrm{m} / 0.5-3)$ and microscopical punctures $(5 \mu \mathrm{m})$ (vs dense and coarse punctures separated by $0.5-1$ puncture diameter).

\section{Etymology}

The specific epithet is named after the Indian state Uttar Pradesh, the type locality of this species.

\section{Type material}

\section{Holotype}

INDIA • O; Uttar Pradesh bor., Karnaprayag env.; [30²7' N, 79²5' E]; 770 m a.s.1.; 19-21 Jul. 1994; M. Valenta leg.; PCMS/OLBL.

\section{Description}

Female (holotype, Fig. 21)

MEASUREMENTS. Total body length $10.5 \mathrm{~mm}$.

HEAD (Fig. 22B-C). Black; transverse, ca 1.25 times as wide as long; lateral preoccipital carina present, well-developed; vertex elevated, distance from top of head to upper margin of lateral ocellus ca 1.5 ocellar diameter as seen in frontal view and ca two diameters as seen in dorsal view; mandibles bi-dentate; F1 0.5 times as long as wide; F2 0.7 times as long as wide; F3 1.1 times as long as wide; supraclypeal area bulging; frons and paraocular area mostly with confluent punctures; ocello-ocular area densely punctate with confluent punctures and punctures separated by at most a half puncture diameter $(30-50 \mu \mathrm{m})$; clypeus sparser punctate with shiny, smooth interspaces equal to at most a puncture diameter; paraocular area and gena with dense plumose setae, obscuring integument.

Mesosoma. Mesosoma (including legs) black; wings with strong brownish darkening and metallic violet luster; stigma and veins dark brown; hind wing with the angle between basal $(M)$ and cubital $(\mathrm{Cu})$ veins ca $70^{\circ}$, costal margin with nine hamuli; mesoscutum and mesoscutellum (Fig. 22A) densely and coarsely punctate $(50-100 \mu \mathrm{m})$, with confluent punctures peripherally and medially with punctures separated by at most a puncture diameter; hypoepimeral area and mesepisternum coarsely reticulaterugose; propodeal triangle (metapostnotum) roughly reticulate-rugose (sculpture forming 2-3 rows of large deep cells).

Metasoma. Metasomal T1 on disc with coarse punctures $(15-25 \mu \mathrm{m})$ separated by $0.5-3$ puncture diameters and laterally with numerous tiny punctures (ca $5 \mu \mathrm{m}$ ) between them, marginal zone sparsely and finely punctate, impunctate along posterior margin (Fig. 22E); remainder of terga similarly punctate, but denser and with impunctate marginal zones (Fig. 22D); pygidial plate dull, as wide as metabasitarsus; T1-T4 red; sterna finely tessellate to smooth with coarse shallow setal pores.

\section{Male}

Unknown.

\section{Distribution}

Only known from the holotype.

\section{Discussion}

In total, 26 species of Sphecodes are now recorded from the Himalayas (Table 1), and 16 of these are distributed beyond the studied region. The Himalayan mountain system is traditionally considered to 
be the boundary between the Palaearctic and the Oriental regions. This feature is reflected in the mixed composition of the fauna of the genus in this region: the Sphecodes fauna of the Himalayas includes eight Palaearctic, eight Oriental and ten endemic species.

The Himalayan fauna of Sphecodes has a high degree of endemicity (38\%), but this phenomenon may be in part due to an incomplete knowledge of the distribution of the Sphecodes fauna. Illustrating this, one half of these endemic species (S. almoransis, S. dissimilandus, S. setiger, S. shillongensis, S. perplexus) are known only from their type localities.

The majority of the Palaearctic Himalayan Sphecodes assemblage is composed of widespread TransPalaearctic or Eurasian species. Only the range of S. hakkariensis is otherwise small, limited to mountainous areas of Turkey and Tajikistan. Interestingly, Sphecodes cameronii is an inter-realm species, distributed from Turkey and Central Asia to India (Pali) and Sri Lanka. All Palaearctic Himalayan species occur in the western to central parts of the Himalayas; moreover, three of them (S. alternatus, S. intermedius, S. hakkariensis) in the studied territory are known from Tajikistan (Gorno-Badakhshan Province) only.

The Oriental Himalayan species of Sphecodes are distributed from the Himalayas to Southeast Asia and China, where they are mainly distributed in mountainous areas. In the Himalayas, these species occur in the central and eastern parts. The ranges of Sphecodes kershawi and S. binghami, which are distributed all the way to Malaysia, are the most widespread among Oriental species occurring in the Himalayas.

\section{Acknowledgements}

We are grateful to Fritz Gusenleitner (OLBL) for help during our visit to Austria, Michael Orr (Beijing, China) for checking the English grammar, and David Notton (NHMUK) for assisting during work in the NHMUK Collection and providing photos of type specimens. We thank Maximilian Schwarz (Ansfelden, Austria), Esther Ockermueller (OLBL), James Hogan (OUMNH), Michael Ohl and Viola Richter (ZMHB) for providing Sphecodes specimens. We also thank Gavin Broad and one anonymous reviewer for their comments and suggestions to streamline and improve the manuscript. This investigation was supported by the Russian Funds for Basic Research (grant numbers 19-04-00027 and 20-54-44014) and the state research project AAAA-A19-119020690101-6.

\section{References}

Ågren L. \& Svensson B. 1982. Flagellar sensilla on Sphecodes bees (Hymenoptera, Halictidae). Zoologica Scripta 11: 45-54. https://doi.org/10.1111/j.1463-6409.1982.tb00517.x

Ascher J.S. \& Pickering J. 2020. Discover Life bee species guide and world checklist (Hymenoptera: Apoidea: Anthophila). Available from $\mathrm{http} / / \mathrm{www}$.discoverlife.org $/ \mathrm{mp} / 20 \mathrm{q}$ ?guide=Apoidea_species [accessed 20 Apr. 2020].

Astafurova Yu.V. \& Proshchalykin M.Yu. 2014. The bees of the genus Sphecodes Latreille 1804 of the Russian Far East, with key to species (Hymenoptera: Apoidea: Halictidae). Zootaxa 3887 (5): 501-528. https://doi.org/10.11646/zootaxa.3887.5.1

Astafurova Yu.V. \& Proshchalykin M.Yu. 2015a. Bees of the genus Sphecodes Latreille 1804 of Siberia, with a key to species (Hymenoptera: Apoidea: Halictidae). Zootaxa 4052 (1): 65-95.

https://doi.org/10.11646/zootaxa.4052.1.3

Astafurova Yu.V. \& Proshchalykin M.Yu. 2015b. New and little known bees of the genus Sphecodes Latreille (Hymenoptera: Halictidae) from Mongolia. Far Eastern Entomologist 289: 1-9. 
Astafurova Yu.V. \& Proshchalykin M.Yu. 2015c. The bees of the genus Sphecodes Latreille, 1804 (Hymenoptera: Halictidae) of the Eastern Palaearctic Region. Proceedings of the Russian Entomological Society 86 (2): 17-21. [In Russian.]

Astafurova Yu.V. \& Proshchalykin M.Yu. 2016a. To the knowledge of the genus Sphecodes Latreille (Hymenoptera: Halictidae) of Caucasus. Euroasian Entomological Journal 15 (Supl. 1): 15-19.

Astafurova Yu.V. \& Proshchalykin M.Yu. 2016b. The bees of the genus Sphecodes Latreille (Hymenoptera: Halictidae) of the European part of Russia. Far Eastern Entomologist 321: 1-21.

Astafurova Yu.V. \& Proshchalykin M.Yu. 2017a. To the knowledge of the Sphecodes hyalinatus Hagens species-group (Hymenoptera, Halictidae). Entomological Review 97 (5): 664-671.

https://doi.org/10.1134/S0013873817050104

Astafurova Yu.V. \& Proshchalykin M.Yu. 2017b. The genus Sphecodes Latreille 1804 (Hymenoptera: Apoidea: Halictidae) in Central Asia. Zootaxa 4324 (2): 249-284.

https://doi.org/10.11646/zootaxa.4324.2.3

Astafurova Yu.V. \& Proshchalykin M.Yu. 2018. A new species of a bee of the genus Sphecodes Latreille (Hymenoptera, Halictidae) from Kazakhstan. Entomological Review 98 (6): 743-747.

https://doi.org/10.1134/S0013873817060118

Astafurova Yu.V., Proshchalykin M.Yu. \& Schwarz M. 2015. New data on the genus Sphecodes Latreille (Hymenoptera: Halictidae) from Mongolia. Far Eastern Entomologist 302: 1-9.

Astafurova Yu.V., Proshchalykin M.Yu. \& Engel M.S. 2018a. The cuckoo bee genus Sphecodes Latreille, 1804 in Kazakhstan (Hymenoptera: Halictidae). Far Eastern Entomologist 369: 1-47.

https://doi.org/10.25221/fee.369.1

Astafurova Yu.V., Proshchalykin M.Yu., Niu Z.-Q. \& Zhu C.-D. 2018b. New records of bees of the genus Sphecodes Latreille in the Palaearctic part of China (Hymenoptera, Halictidae). ZooKeys 792: 15-44. https://doi.org/10.3897/zookeys.792.28042

Astafurova Yu.V., Proshchalykin M.Yu. \& Schwarz M. 2018c. New and little known bees of the genus Sphecodes Latreille, 1804 (Hymenoptera: Apoidea: Halictidae) from Central Asia. Zootaxa 4441 (1): 76-88. https://doi.org/10.11646/zootaxa.4441.1.4

Astafurova Yu.V., Proshchalykin M.Yu. \& Schwarz M. 2018d.The cuckoo bee genus Sphecodes Latreille, 1804 (Hymenoptera: Halictidae) in Iran. Journal of Hymenoptera Research 66: 39-53.

https://doi.org/10.3897/jhr.66.29269

Astafurova Yu.V., Proshchalykin M.Yu. \& Schwarz M. 2019. The distribution of the genus Sphecodes Latreille (Hymenoptera, Halictidae) of the Arabian Peninsula and surrounding countries with description of hitherto unknown female of S. atlanticus Warncke, 1992 and male of S. dathei Schwarz, 2010. ZooKeys 872: 13-40. https://doi.org/10.3897/zookeys.872.35361

Astafurova Yu.V., Proshchalykin M.Yu., Niu Z.-Q., Orr M.C. \& Zhu C.-D. 2020a. New and little-known bees of the genus Sphecodes Latreille, 1804 (Hymenoptera, Apoidea, Halictidae) from Southern and South-Western China. Journal of Hymenoptera Research 79: 145-162.

https://doi.org/10.3897/jhr.79.57276

Astafurova Yu.V., Proshchalykin M.Yu. \& Schwarz M. 2020b. New and little-known species of the genus Sphecodes Latreille (Hymenoptera, Halictidae) from Southeast Asia. ZooKeys 937: 31-88. https://doi.org/10.3897/zookeys.937.51708

Bingham C.T. 1897. The Fauna of British India including Ceylon and Burma, Hymenoptera, Vol. I. Wasps and Bees. Taylor and Francis, London. https://doi.org/10.5962/bhl.title.100738 
Bingham C.T. 1898. On some new species of Indian Hymenoptera. Journal of the Bombay Natural History Society 12 (1): 115-130.

Blüthgen P. 1924. Beiträge zur Systematik derBienengattung Sphecodes Latr. II.Deutsche Entomologische Zeitschrift 1924: 457-516.

Blüthgen P. 1927. Beiträge zur Systematik der Bienengattung Sphecodes Latr. III. Zoologische Jahrbucher, Abteilung fur Systematik, Geographie und Biologie der Tiere 53 (1/3): 23-112.

Blüthgen P. 1938. Neue Halictidi aus Zypern. Konowia 16: 41-54.

Bogusch P. \& Straka J. 2012. Review and identification of the cuckoo bees of central Europe (Hymenoptera: Halictidae: Sphecodes). Zootaxa 3311 (1): 1-41.

https://doi.org/10.11646/zootaxa.3311.1.1

Cameron P. 1897. Hymenoptera orientalia, or contributions to a knowledge of the Hymenoptera of the Oriental Zoological Region. Part V. Memoirs, Manchester Literary and Philosophical Society 41 (4): $1-144$.

Christ J.L. 1791. Naturgeschichte, Klassifikation und Nomenklatur der Insekten vom Bienen, Wespen und Ameisengeschlecht: als der fünften Klasse fünfte Ordnung des Linneischen Natursystems von den Insekten, Hymenoptera: mit häutigen Flügeln. Hermannischen Buchhandlung, Frankfurt am Main. https://doi.org/10.5962/bhl.title.87724

Cockerell T.D.A. 1916. Descriptions and records of bees-LXXII. Annals and Magazine of Natural History Series 88 (17): 428-435. https://doi.org/10.1080/00222931608693809

Cockerell T.D.A. 1921. Descriptions and records of bees-XCI. Annals and Magazine of Natural History Series 98 (45): 359-368. https://doi.org/10.1080/00222932108632595

Cockerell T.D.A. 1930. Descriptions and records of bees-CXXII. Annals and Magazine of Natural History Series 105 (25): 156-163. https://doi.org/10.1080/00222933008673114

Cockerell T.D.A. 1931a. Descriptions and records of bees-CXXVII. Annals and Magazine of Natural History Series 1010 (7): 344- 351. https://doi.org/10.1080/00222933108673321

Cockerell T.D.A. 1931b. Descriptions and records of bees-CXXIX. Annals and Magazine of Natural History Series 1010 (8): 411-418. https://doi.org/10.1080/00222933108673413

Cockerell T.D.A. 1937. Siberian bees of the genera Halictus, Sphecodes, and Hylaeus. American Museum Novitates 949: 1- 6. Available from http://hdl.handle.net/2246/2186 [accessed 14 Dec. 2020].

Dalla Torre C.G. de 1896. Catalogus Hymenopterorum hucusque descriptorum systematicus et synonymicus. Vol. X. Apidae (Anthophila). Engelmann, Leipzig [Lipsiae].

Dalla Torre K.W. 1887. Die Apiden Tirols. (Fortsetzung von 1873). Zeitschrift des Ferdinandeums 21 (3): 159-196.

Dar S.A. \& Wani A.R. 2018. Cleptoparasitic behaviour of Sphecodes tantalus Nurse (1903) on Lasioglossum marginatum (Brulle) in Kashmir. Indian Journal of Entomology 80 (4): 1431-1435. https://doi.org/10.5958/0974-8172.2018.00339.5

Dar S.A., Malik T.-U.-H., Dar S.H., Nissar M., Wani R.A., Kandoo A.A. \& Rather B.A. 2018. Foraging pattern of Lasioglossum (Hymenoptera: Halictidae) species on Himalayan indigo (Fabaceae) in Kashmir. The Pharma Innovation Journal 7 (5): 530-533.

Engel M.S. 2001. A monograph of the Baltic amber bees and evolution of the Apoidea (Hymenoptera). Bulletin of the American Museum of Natural History 259: 1-192.

https://doi.org/10.1206/0003-0090(2001)259<0001:AMOTBA>2.0.CO;2 
Fabricius J.C. 1793. Entomologia Systematica Emendata et Aucta, Secundum Classes, Ordines, Genera, Species Adjectis Synonymis, Locis, Observationibus, Descriptionibus. Volume 2. Proft, Copenhagen [Hafniae]. https://doi.org/10.5962/bhl.title.36532

Fabricius J.C. 1804. Systema Piezatorum Secundum Ordines, Genera, Species Adjectis Synonymis, Locis, Observationibus, Descriptionibus. Reichard, Braunschweig [Brunsvigae].

https://doi.org/10.5962/bhl.title.10490

Füessly J.C. 1775. Verzeichnis der ihm bekannten Schweizerischen Insecten mit einer ausgemahlten Kupfertafel: nebst der Ankündigung eines neuen Insecten Werks. H. Steiner, Zürich.

https://doi.org/10.5962/bhl.title.65772

Germar E.F. 1819. Fauna Insectorum Europae. Vol. 5. Kümmel, Halle an der Saale [Halae].

Gistel J. 1857. Achthundert und zwanzig neue oder unbeschchriebene wirbellose Thiere. Vacuna 2: 513-606.

Gupta R.K. 2013. Apoidea (Insecta: Hymenoptera) of Uttarakhand state (India): a checklist with synonymies and distribution record. Journal of Environment and Bio-Sciences 27 (1): 57-70.

Gupta R.K. \& Saini J. 2018. A new species of clepto-parasitic bee genus Sphecodes Latreille (Insecta: Hymenoptera) from Almora, Uttarakhand, India. Journal of Environment and Bio-Sciences 32 (1): 113114.

Harris R.A. 1979. A glossary of surface sculpturing. Occasional papers of the Bureau of Entomology of the California Department of Agriculture 28: 1-31. https://doi.org/10.5281/zenodo.26215

Illiger K. 1806. William Kirbys Familien der bienenartigen Insekten mit Zusätzen, Nachweisungen und Bemerkungen. Magazin für Insektenkunde 5: 28-175.

Inoka W.A., Karunaratne P., Edirisinghe J.P. \& Pauly A. 2005. An Updated Checklist of Bees of Sri Lanka with new Records. National Science Foundation of Sri Lanka, Colombo.

International Commission on Zoological Nomenclature (ICZN) 1999. International Code of Zoological Nomenclature. 4th Edition. ITZN, London.

Kirby W. 1802. Monographia Apum Angliae; or, an Attempt to Divide into their Natural Genera and Families, such Species of the Linnean Genus Apis as have been Discovered in England. Vol. 2. J. Raw, privately published, Ipswich. https://doi.org/10.5962/bhl.title.10346

Lepeletier de Saint Fargeau A.L.M. 1841. Histoire naturelle des Insectes. Hyménoptères. Volume. 2. Roret, Paris. https://doi.org/10.5962/bhl.title.10345

Linnaeus C. 1758. Systema Naturae per Regna Tria Naturae Secundum Classes, Ordines, Genera, Species, cum Characteribus, Differentiis, Synonymis, Locis. Vol. I. Editio X. Laurentii Salvii, Stockholm [Holmiae]. https://doi.org/10.5962/bhl.title.542

Meyer R. 1920. Apidae-Sphecodinae. Archiv für Naturgeschichte, Abt. A 1919 (1): 79-160; (2): 161242.

Meyer R. 1922. Nachtrag I zur Bienengattung Sphecodes Latr. Archiv für Naturgeschichte 88A (8): $165-174$.

Meyer R. 1925. Zur Bienengattung Sphecodes. Archiv für Naturgeschichte 90A (12): 1-12.

Michener C.D. 2007. The Bees of the World. $2^{\text {nd }}$ Ed. The Johns Hopkins University Press, Baltimore.

Morawitz F. 1876. A Travel to Turkestan by the Member-Founder of the Society A.P. Fedtschenko, accomplished from the Imperial Society of Naturalists, Anthropologists, and Ethnographists on a Commission from the General-Governor of Turkestan K.P. von Kaufmann (Issue 13). Vol. II. 
Zoogeographical Investigations. Pt. V. (Division 7). Bees (Mellifera). Pt. II [Andrenidae]. Izvestiya Imperatorskogo Obshchestva Lyubiteley Estestvoznaniya, Anthropologii i Ethnografii 21 (3): 161-303. [In Russian.]

Natural History Museum 2014. Dataset: Collection specimens. Resource: Specimens. Natural History Museum Data Portal. Available from https://data.nhm.ac.uk [accessed 15 Aug. 2017]. https://doi.org/10.5519/0002965

Nurse C.G. 1903. New species of Indian aculeate Hymenoptera. Annals and Magazine of Natural History Series 7 11: 393-403, 511-526, 528-549. https://doi.org/10.1080/00222930308678811

Olivier A.G. 1789. Encyclopedie méthodique, Dictionnaire des Insectes. Vol. 4. Panckoucke, Paris.

Özbek H., Bogusch P. \& Straka J. 2015. A contribution to the kleptoparasitic bees of Turkey: Part I., the genus Sphecodes Latreille (Hymenoptera: Halictidae). Turkish Journal of Zoology 39 (6): 1095-1109. https://doi.org/10.3906/zoo-1501-43

Perkins R.C.L. 1921. Two new species of bees of the genus Sphecodes. Entomologist's Monthly Magazine 57: 9-11.

Pittioni B. 1950. Hymenoptera Aculeata I. On the insectfauna of Cyprus. Results of the Expedition of 1939 by Harald, Hakan and P. H. Lindberg. Commentationes Biologicae, Societas Scientiarum Fennica 10: 1-94.

Rajkumar M.B. \& Dey D. 2016. Identification and description of Indian parasitic bee genus Sphecodes Latreille 1804, (Halictidae: Hymenoptera). Journal of Applied and Natural Science 8 (4): 1839-1849. https://doi.org/10.31018/jans.v8i4.1051

Saini M.S. \& Rathor V.S. 2012. A species checklist of family Halictidae (Hymenoptera: Apoidea) along with keys to its subfamilies, genera \& subgenera from India. International Journal of Environmental Sciences 3 (1): 134-166.

Sichel J. 1865. Études hyménoptèrologiques. Annales de la Société entomologique de France 4 (5): 331-492.

Smith F. 1845. Descriptions of the British species of bees belonging to the genus Sphecodes of Latreille. Zoologist 3: 1011-1015.

Smith F. 1848. Descriptions of the British species of bees belonging to the genus Halictus of Latreille. Zoologist 6: 2209.

Smith F. 1853 Catalogue of Hymenopterous Insects in the Collection of the British Museum. Andrenidae and Apidae. Vol. 1. Printed by order of the Trustees, London. https://doi.org/10.5962/bhl.title.20858

Smith F. 1879. Descriptions of New Species of Hymenoptera in the Collection of the British Museum. British Museum, London. https://doi.org/10.5962/bhl.title.17490

Verhoeff C. 1890. Ein Beitrag zur deutschen Hymenopteren-Fauna. Entomologische Nachrichten 16: 321-336.

Warncke K. 1992. Die westpaläarktischen Arten der Bienengattung Sphecodes Latr. Bericht der Naturforschende Gesellschaft Augsburg 52: 9-64.

Wesmael C. 1835. Observations sur les espèces du genre Sphécode. Bulletin et Annales de la Société royal d'Entomologie de Belgique 2: 279-287. 
Published on: 28 December 2020

Topic editor: Gavin Broad

Topic editor: Nesrine Akkari

Desk editor: Pepe Fernández

Printed versions of all papers are also deposited in the libraries of the institutes that are members of the EJT consortium: Muséum national d'histoire naturelle, Paris, France; Meise Botanic Garden, Belgium; Royal Museum for Central Africa, Tervuren, Belgium; Royal Belgian Institute of Natural Sciences, Brussels, Belgium; Natural History Museum of Denmark, Copenhagen, Denmark; Naturalis Biodiversity Center, Leiden, the Netherlands; Museo Nacional de Ciencias Naturales-CSIC, Madrid, Spain; Real Jardín Botánico de Madrid CSIC, Spain; Zoological Research Museum Alexander Koenig, Bonn, Germany; National Museum, Prague, Czech Republic. 